\title{
The Chemical Homogeneity of Sun-like Stars in the Solar Neighborhood
}

\author{
Megan Bedell ${ }^{1,2}$ (D), Jacob L. Bean ${ }^{2}$ (D) , Jorge Meléndez ${ }^{3}$, Lorenzo Spina ${ }^{3,4}$, Ivan Ramírez ${ }^{5}$, Martin Asplund ${ }^{6}$ (D), Alan Alves-Brito ${ }^{7}$, \\ Leonardo dos Santos ${ }^{3,8}$, Stefan Dreizler ${ }^{9}$, David Yong ${ }^{6}$, TalaWanda Monroe ${ }^{10}$ (D), and Luca Casagrande ${ }^{6}$ (i) \\ ${ }^{1}$ Center for Computational Astrophysics, Flatiron Institute, 162 5th Avenue, New York, NY 10010, USA; mbedell@ flatironinstitute.org \\ ${ }^{2}$ Department of Astronomy and Astrophysics, University of Chicago, 5640 S. Ellis Avenue, Chicago, IL 60637, USA \\ ${ }_{3}^{3}$ Departamento de Astronomia do IAG/USP, Universidade de São Paulo, Rua do Matão 1226, Cidade Universitária, 05508-900 São Paulo, SP, Brazil \\ ${ }^{4}$ Monash Centre for Astrophysics, School of Physics and Astronomy, Monash University, VIC 3800, Australia \\ ${ }^{5}$ Tacoma Community College, 6501 South 19th Street, Tacoma, Washington 98466, USA \\ ${ }^{6}$ Research School of Astronomy and Astrophysics, The Australian National University, Cotter Road, Canberra, ACT 2611, Australia \\ ${ }^{7}$ Universidade Federal do Rio Grande do Sul, Instituto de Física, Av. Bento Gonçalves 9500, Porto Alegre, RS, Brazil \\ ${ }^{8}$ Observatoire de l'Université de Genève, 51 chemin des Maillettes, 1290 Versoix, Switzerland \\ ${ }^{9}$ Institut für Astrophysik, Georg-August-Universität, Friedrich-Hund-Platz 1, D-37077 Göttingen, Germany \\ ${ }^{10}$ Space Telescope Science Institute, 3700 San Martin Drive, Baltimore, MD 21218, USA \\ Received 2018 February 7; revised 2018 August 2; accepted 2018 August 7; published 2018 September 21
}

\begin{abstract}
The compositions of stars are a critical diagnostic tool for many topics in astronomy such as the evolution of our Galaxy, the formation of planets, and the uniqueness of the Sun. Previous spectroscopic measurements indicate a large intrinsic variation in the elemental abundance patterns of stars with similar overall metal content. However, systematic errors arising from inaccuracies in stellar models are known to be a limiting factor in such studies, and thus it is uncertain to what extent the observed diversity of stellar abundance patterns is real. Here we report the abundances of 30 elements with precisions of $2 \%$ for 79 Sun-like stars within 100 pc. Systematic errors are minimized in this study by focusing on solar twin stars and performing a line-by-line differential analysis using high-resolution, high-signal-to-noise spectra. We resolve $[\mathrm{X} / \mathrm{Fe}]$ abundance trends in galactic chemical evolution at precisions of $10^{-3} \mathrm{dex} \mathrm{Gyr}^{-1}$ and reveal that stars with similar ages and metallicities have nearly identical abundance patterns. Contrary to previous results, we find that the ratios of carbon-to-oxygen and magnesium-tosilicon in solar-metallicity stars are homogeneous to within $10 \%$ throughout the solar neighborhood, implying that exoplanets may exhibit much less compositional diversity than previously thought. Finally, we demonstrate that the Sun has a subtle deficiency in refractory material relative to $>80 \%$ of solar twins (at $2 \sigma$ confidence), suggesting a possible signpost for planetary systems like our own.
\end{abstract}

Key words: planets and satellites: general - stars: abundances - stars: solar-type - Sun: abundances - techniques: spectroscopic

Supporting material: machine-readable tables

\section{Introduction}

The photosphere of a Sun-like star acts as a fossil record of the nebular cloud from which the star formed, making spectroscopic measurements of stellar compositions an informative probe of chemical evolution throughout the galaxy. Stellar compositions can also support exoplanet studies: because the star and its planets form side-by-side from the same primordial material, the relative compositions of stars that host different types of planets yield constraints on planet formation processes (e.g., Gonzalez 1997; Fischer \& Valenti 2005; Thiabaud et al. 2015) and may even indicate the detailed physical properties of known planets (e.g., Unterborn et al. 2014; Dorn et al. 2015; Santos et al. 2015; Unterborn \& Panero 2017).

Previous investigations on this topic indicate that stars with similar overall metal content display significant diversity in their elemental abundance patterns (Adibekyan et al. 2012; Bensby et al. 2014; Brewer \& Fischer 2016). This implies a wide range in the possible properties of exoplanets. In particular, the abundance ratios of $\mathrm{C} / \mathrm{O}$ and $\mathrm{Mg} / \mathrm{Si}$ have been found to vary considerably, carrying dramatic consequences for the nature of terrestrial planets formed around these stars (Delgado Mena et al. 2010; Petigura \& Marcy 2011; SuárezAndrés et al. 2018). If, for example, a significant population of stars form from gas with a primordial $\mathrm{C} / \mathrm{O}$ ratio $>0.8$, this could lead to carbon-rich planets, which would carry significant repercussions when interpreting the observed compositions of such planets' atmospheres (see, e.g., Madhusudhan et al. 2011). Similarly, the $\mathrm{Mg} / \mathrm{Si}$ ratio is a key parameter in models of rocky planet interior structure, with a $\mathrm{Mg} / \mathrm{Si}$ ratio significantly below 1 or above 2 leading to non-Earth-like balances of compounds like pyroxine and olivine within the planet and potentially altering its geological processes (Sotin et al. 2007; Carter-Bond et al. 2012).

Beyond individual elemental abundances or ratios, subtle trends across the full set of measured abundances can also carry information relevant to planets. In a differential spectroscopic study of 11 solar twins, Meléndez et al. (2009, hereafter M09) found that the Sun has an unusual abundance pattern characteristic of dust condensation. When comparing the Sun to the average abundance pattern of 11 solar twins, M09 observed a deficit of refractory elements relative to volatiles. This trend was quantified as a slope in abundance $[\mathrm{X} / \mathrm{H}]$ as a function of condensation temperature $\left(T_{\mathrm{C}}\right)$, the temperature at which an element is expected to condense under protoplanetary disk conditions (Lodders 2003). This $T_{\mathrm{C}}$ trend has remained a topic of extensive debate, with some studies reproducing M09's findings and others being unable to confirm its existence for various samples of Sun-like stars (Ramírez et al. 2009, 2010; 
González Hernández et al. 2010, 2013; Gonzalez et al. 2010; Gonzalez 2011; Schuler et al. 2011).

A variety of explanations for $T_{\mathrm{C}}$ trends have been proposed. M09 suggested that the solar $T_{\mathrm{C}}$ trend may arise as a result of rocky planet formation. Chambers (2010) subsequently demonstrated that the Sun's refractory deficit corresponds to 4 Earth masses of terrestrial and chondritic material, confirming the plausibility of this explanation. On the other hand, it is also possible that a positive $T_{\mathrm{C}}$ slope could be a signature of latestage accretion of planetary material (e.g., Ramírez et al. 2011; Spina et al. 2015; Oh et al. 2018), implying that most solar twins have ingested planets in the past. Additionally, while most studies have focused on potential links between $T_{\mathrm{C}}$ trends and planet formation, it is also likely that $T_{\mathrm{C}}$ behavior is affected by galactic chemical evolution (GCE) and thereby correlates with stellar age (Adibekyan et al. 2014; Nissen 2015; Spina et al. 2016a). Alternative causes of the Sun's unusual $T_{\mathrm{C}}$ trend include gas-dust segregation in the protoplanetary disk and dust cleansing in the primordial nebula (Önehag et al. 2014; Gaidos 2015).

It is clear that the detailed abundances of stars, when known at a sufficient level of precision, can strongly inform our understanding of planet formation and the nature of exoplanetary systems. However, systematic errors arising from inaccuracies in stellar models, uncertainties in GCE, and small sample sizes are known to be limiting factors in such studies, and thus the robustness of previous results is highly debated (Asplund et al. 2009; Fortney 2012; Adibekyan et al. 2014; Nissen 2015; Hinkel et al. 2016).

In this work, we focus on solar twin stars to minimize stellar model-driven biases and achieve maximum-precision abundance measurements. These objects are a subset of mainsequence stars with effective temperatures, surface gravities, and overall metallicities very close to the solar values, thus ensuring that the photospheric conditions under which their spectra originate are as similar as possible to the Sun's. As a result, relatively little input from stellar atmospheric models is needed to measure spectroscopic abundances in these stars relative to the solar composition provided that these measurements are done in a strictly differential way. This technique enables us to sidestep the systematic biases introduced by models and achieve abundance measurements with precisions below the level of 0.01 dex, or $2 \%$ (Bedell et al. 2014).

We present precise abundance measurements for 16 light $(Z \leqslant 30)$ elements in 79 stars. We couple these new measurements with the stellar ages and heavy-element abundances for the same sample presented in Spina et al. (2018). The resulting set of abundances for 30 elements across a uniformly analyzed sample of many Sun-like stars enables the investigation at an unprecedentedly detailed level of the behavior of stellar compositions as a function of age, the range of relative concentrations of key planet-forming elements, and the spread of abundance- $T_{\mathrm{C}}$ trend behavior.

\section{Data}

Solar twin stars for this study were selected from a variety of sources including color-based dedicated solar twin searches (Meléndez \& Ramírez 2007; Meléndez et al. 2009; Ramírez et al. 2009) as well as twins identified in past surveys (Valenti \& Fischer 2005; Baumann et al. 2010; Bensby et al. 2014). The general selection criteria used were effective temperature $\left(T_{\text {eff }}\right)$ within $100 \mathrm{~K}$ of solar; surface gravity $(\log g)$ within 0.1 dex of solar; and metallicity (taken as the iron abundance $[\mathrm{Fe} / \mathrm{H}]$ ) within 0.1 dex of solar. In some cases our final derived parameters (see Section 3.1) are slightly outside of these bounds.

To achieve sufficient signal-to-noise for high-precision abundance work, we stacked $\geqslant 50$ observations for each star. All spectra were taken with the High Accuracy Radial velocity Planet Searcher (HARPS) spectrograph on the 3.6 meter telescope of the European Southern Observatory (ESO), located at La Silla Observatory in Chile (Mayor et al. 2003). HARPS is an ultra-stable echelle spectrograph with resolving power $R=115,000$ and wavelength coverage between 378-691 nm. A majority of the selected sample were observed by us in the course of a large ESO observing program on HARPS (Meléndez et al. 2015). Other stars had a sufficient number of publicly available spectra in the online ESO Science Archive Facility. ${ }^{11}$

The HARPS pipeline provides extracted one-dimensional spectra and radial velocity information automatically. Using these data products, we Doppler-corrected, continuum normalized, and stacked all spectra for a given star to create a single composite spectrum with a very high-signal-to-noise ratio $(\mathrm{S} / \mathrm{N})$. This procedure was done slightly differently for the blue $(378<\lambda<530 \mathrm{~nm})$ and red $(533<\lambda<691 \mathrm{~nm})$ CCD chips. The blue chips were automatically continuum normalized using a twentieth-order polynomial fit in IRAF's onedspec.continuum module. ${ }^{12}$ The red chips, which are more strongly affected by wide bands of telluric absorption lines, were hand-fit in interactive mode. In either case, efforts were made to ensure that all spectra were normalized similarly across stars, since our differential equivalent width technique is more sensitive to star-to-star differences than it is to absolute continuum slopes.

Combined spectra for a total of 79 targets were created in this manner. An additional three stars (HIP 19911, HIP 67620, and HIP 103983) were included in the initial solar twin sample, but were later dropped due to spectral contamination by a nearby companion (dos Santos et al. 2017). The combined spectra of the remaining stars varies in quality depending on the brightness of the target and the number of HARPS spectra observed, but on average an $\mathrm{S} / \mathrm{N}$ of approximately $800 \mathrm{pix}^{-1}$ at $600 \mathrm{~nm}$ is achieved. The minimum and maximum $\mathrm{S} / \mathrm{N}$ in the sample are 300 and $1800 \mathrm{pix}^{-1}$.

The solar reference spectrum used in this work was created by combining multiple exposures of sunlight reflected from the asteroid Vesta. It was continuum-normalized in the same manner as the target spectra and has $\mathrm{S} / \mathrm{N} \sim 1300 \mathrm{pix}^{-1}$ at $600 \mathrm{~nm}$.

Spectra previously obtained with the MIKE spectrograph (Bernstein et al. 2003) and analyzed in Ramírez et al. (2014) were also used in some parts of this analysis. These spectra have $\mathrm{S} / \mathrm{N} \sim 400 \mathrm{pix}^{-1}$ at $600 \mathrm{~nm}$, resolution $R=83,000-65,000$ (on blue/red CCDs), and wavelength coverage between 320 and $1000 \mathrm{~nm}$.

\footnotetext{
${ }^{11}$ Data were used from ESO programme IDs 072.C-0488, 074.C-0364, 075. C-0202, 075.C-0332, 076.C-0155, 077.C-0364, 088.C-0323, 089.C-0415, 089.C-0732, 090.C-0421, 091.C-0034, 091.C-0936, 092.C-0721, 093.C0409, 095.C-0551, 096.C-0499, 097.C-0090, 097.C-0571, 097.C-0948, 183. C-0972, 183.D-0729, 185.D-0056, 188.C-0265, 192.C-0224, 192.C-0852, 289.C-5053, 292.C-5004, and 60.A-9036.

12 IRAF is distributed by the National Optical Astronomy Observatory, which is operated by the Association of Universities for Research in Astronomy (AURA) under cooperative agreement with the National Science Foundation.
} 


\section{Analysis}

\subsection{Stellar Parameters and Abundances}

We use a strictly differential line-by-line equivalent width technique, as detailed in Bedell et al. (2014), to obtain stellar parameters and abundances. In brief, we measure equivalent widths using IRAF's splot module for each line of interest. We then compute the corresponding abundance for each line using MOOG with stellar models from the Kurucz ATLAS9 grid (Sneden 1973; Castelli \& Kurucz 2004). We subtract the calculated abundances of the solar reference spectrum from each target spectrum before combining lines of a given species to yield a final estimate of the abundance.

As presented in Spina et al. (2018), we first measured a set of $98 \mathrm{Fe} \mathrm{I}$ and $17 \mathrm{Fe}$ II lines and used these to identify the optimal stellar model parameters (effective temperature $T_{\text {eff }}$, surface gravity $\log g$, metallicity $[\mathrm{Fe} / \mathrm{H}]$, and microturbulent velocity $v_{t}$ ). This is done by imposing several criteria on the resulting differential iron abundances of a given star and sampling model parameter space until these criteria are fulfilled. These criteria consist of the following: (a) slope of the abundance versus reduced equivalent width relation consistent with zero; (b) slope of the abundance versus line excitation potential relation consistent with zero; (c) average abundance of Fe I consistent with that of Fe II; and (d) derived iron abundance consistent with the input metallicity of the model. The scatter in abundances from the sample of Fe I and Fe II lines are propagated through to define a $1 \sigma$ uncertainty range on the derived parameters. The procedure of solving for stellar parameters and their uncertainties was performed automatically using the python package $q^{2}$ (Ramírez et al. 2014).

The resulting stellar parameters for the full 79-star sample are shown in Figure 1. A complete list of the parameters and an analysis of their precision can be found in Spina et al. (2018).

Using the same differential equivalent width method, we measured the abundances of 17 additional elements in each spectrum: C, O, Na, Mg, Al, Si, S, Ca, Sc, Ti, V, Cr, Mn, Co, $\mathrm{Ni}, \mathrm{Cu}$, and $\mathrm{Zn}$. The line list was adapted from previous works (Bedell et al. 2014; Ramírez et al. 2014). Hyperfine structure was considered for the $\mathrm{V}, \mathrm{Mn}, \mathrm{Co}$, and $\mathrm{Cu}$ lines using MOOG blends. Four elements were measured in multiple species: C I and $\mathrm{CH}, \mathrm{Sc} \mathrm{I}$ and Sc II, Ti I and Ti II, and Cr I and Cr II. Oxygen was measured from the MIKE spectra rather than HARPS, as further infrared coverage was needed for the OI triplet. The oxygen abundances were then corrected for departures from local thermal equilibrium (Ramírez et al. 2013). When selecting lines, we preferred relatively strong and well-isolated lines without too many blends, which can be fit with a single Gaussian to determine the equivalent width. For some species, however, such unblended lines are not readily available. In these cases, the equivalent width was measured either by fitting a blend of multiple Gaussians (if the centers of the blended lines are sufficiently separated) or by fitting a single Gaussian while avoiding one contaminated wing. The $\mathrm{S}$ I and $\mathrm{CH}$ lines used in this analysis were typically composed of multiple closely grouped weak lines of the same species; we found that fitting a single broad Gaussian to the entire set yielded acceptable results.

Final abundances for each element were obtained by taking an average over the measured abundances for all lines (including all measured species of a given element). Uncertainties on these abundances come from the standard error on
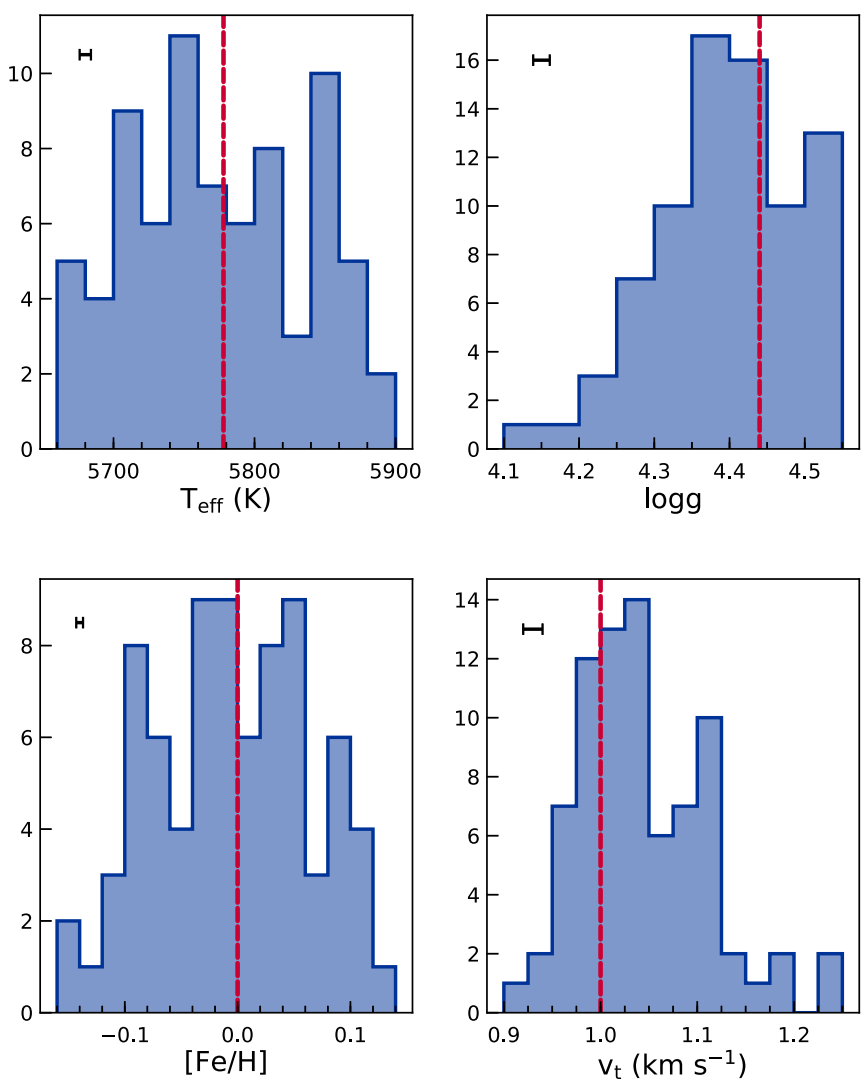

Figure 1. Distribution of fundamental stellar parameters (effective temperature $T_{\text {eff }}$, surface gravity $\log g$, metallicity $[\mathrm{Fe} / \mathrm{H}]$, and microturbulent velocity $v_{t}$ ) for the 79-star sample. The targets fall within a relatively narrow range around the solar values (vertical dashed lines). The median uncertainty for each quantity is shown as a single $1 \sigma$ error interval in the upper left corner of the panel.

the mean, added in quadrature with the propagated effects of the uncertainties on the stellar model parameters. This analysis is automated in the $q^{2}$ package and can be fully reproduced using the provided list of measured equivalent widths (Table 1). The resulting differential abundances are provided in Table 2.

In the following analysis, we also use abundance measurements of 12 heavy elements ( $\mathrm{Sr}, \mathrm{Y}, \mathrm{Zr}, \mathrm{Ba}, \mathrm{La}, \mathrm{Ce}, \mathrm{Pr}, \mathrm{Nd}, \mathrm{Sm}$, $\mathrm{Eu}, \mathrm{Gd}$, and Dy). These abundances are adopted from Spina et al. (2018), which uses the same combined HARPS spectra and the same strictly differential equivalent width method of abundance determination. The resulting data set of 30 elemental abundances in 79 Sun-like stars is the most extensive set of extremely precise differential abundances produced to date.

The stellar sample considered here overlaps with that of Nissen $(2015,2016)$, who also achieve precise abundances using a similar but independently performed analysis of 21 solar twins. Using the 14 common stars to compare the two data sets, we find extremely good agreement, suggesting that both works do achieve sub-0.01 dex precision in the abundances of most elements (Figure 2). Of the 18 elements measured by Nissen (2015) or Nissen (2016; C, O, Na, Mg, Al, $\mathrm{Si}, \mathrm{S}, \mathrm{Ca}, \mathrm{Sc}, \mathrm{Ti}, \mathrm{Cr}, \mathrm{Mn}, \mathrm{Fe}, \mathrm{Ni}, \mathrm{Cu}, \mathrm{Zn}, \mathrm{Y}$, and Ba), most display a mean difference and a standard deviation of less than 0.01 dex between the two samples. The elements with the greatest systematic difference between the two samples are $\mathrm{O}$, where this work finds an offset of +0.015 dex relative to 
Table 1

Contents of Equivalent Width Measurements Table

\begin{tabular}{|c|c|c|c|}
\hline Num & Name & Units & Notes \\
\hline 1 & Wavelength & $\AA$ & Rest wavelength of line \\
\hline 2 & Species & $\cdots$ & Species identifier \\
\hline 3 & $\mathrm{EP}$ & $\mathrm{eV}$ & Excitation potential \\
\hline 4 & $\log (g f)$ & $\operatorname{dex}$ & Log of the oscillator strength \\
\hline 5 & HIP 10175 & $\mathrm{~m} \AA$ & $\ldots$ \\
\hline 6 & HIP 101905 & $\mathrm{m \AA}$ & $\cdots$ \\
\hline 7 & HIP 102040 & $\mathrm{~m} \AA$ & $\ldots$ \\
\hline 8 & HIP 102152 & $\mathrm{m \AA}$ & $\cdots$ \\
\hline 9 & HIP 10303 & $\mathrm{m \AA}$ & $\cdots$ \\
\hline 10 & HIP 104045 & $\mathrm{~m} \AA$ & $\ldots$ \\
\hline 11 & HIP 105184 & $\mathrm{~m} \AA$ & $\ldots$ \\
\hline 12 & HIP 108158 & $\mathrm{mÅ}$ & $\cdots$ \\
\hline 13 & HIP 108468 & $\mathrm{~mA}$ & $\cdots$ \\
\hline 14 & HIP 109821 & $\mathrm{m \AA}$ & $\cdots$ \\
\hline 15 & HIP 114328 & $\mathrm{m \AA}$ & $\cdots$ \\
\hline 16 & HIP 114615 & $\mathrm{m \AA}$ & $\ldots$ \\
\hline 17 & HIP 115577 & $\mathrm{~m} \AA$ & $\ldots$ \\
\hline 18 & HIP 116906 & $\mathrm{m \AA}$ & $\cdots$ \\
\hline 19 & HIP 117367 & $\mathrm{mÅ}$ & $\cdots$ \\
\hline 20 & HIP 118115 & $\mathrm{~m} \AA$ & $\ldots$ \\
\hline 21 & HIP 11915 & $\mathrm{m \AA}$ & $\cdots$ \\
\hline 22 & HIP 14501 & $\mathrm{~m} \AA$ & $\ldots$ \\
\hline 23 & HIP 14614 & $\mathrm{~m} \AA$ & $\ldots$ \\
\hline 24 & HIP 15527 & $\mathrm{mÅ}$ & $\cdots$ \\
\hline 25 & HIP 18844 & $\mathrm{~m} \AA$ & $\ldots$ \\
\hline 26 & HIP 1954 & $\mathrm{~mA}$ & $\ldots$ \\
\hline 27 & HIP 22263 & $\mathrm{~mA}$ & $\ldots$ \\
\hline 28 & HIP 25670 & $\mathrm{~mA}$ & $\ldots$ \\
\hline 29 & HIP 28066 & $\mathrm{~m} \AA$ & $\ldots$ \\
\hline 30 & HIP 29432 & $\mathrm{m \AA}$ & $\ldots$ \\
\hline 31 & HIP 29525 & $\mathrm{mÅ}$ & $\cdots$ \\
\hline 32 & HIP 30037 & $\mathrm{~m} \AA$ & $\ldots$ \\
\hline 33 & HIP 30158 & $\mathrm{m \AA}$ & $\ldots$ \\
\hline 34 & HIP 30476 & $\mathrm{~m} \AA$ & $\ldots$ \\
\hline 35 & HIP 30502 & $\mathrm{~m} \AA$ & $\ldots$ \\
\hline 36 & HIP 3203 & $\mathrm{mÅ}$ & $\cdots$ \\
\hline 37 & HIP 33094 & $\mathrm{~m} \AA$ & $\ldots$ \\
\hline 38 & HIP 34511 & $\mathrm{~mA}$ & $\ldots$ \\
\hline 39 & HIP 36512 & $\mathrm{~m} \AA$ & $\ldots$ \\
\hline 40 & HIP 36515 & $\mathrm{m \AA}$ & $\ldots$ \\
\hline 41 & HIP 38072 & $\mathrm{~m} \AA$ & $\ldots$ \\
\hline 42 & HIP 40133 & $\mathrm{~m} \AA$ & $\ldots$ \\
\hline 43 & HIP 41317 & $\mathrm{mÅ}$ & $\cdots$ \\
\hline 44 & HIP 42333 & $\mathrm{~m} \AA$ & $\ldots$ \\
\hline 45 & HIP 43297 & $\mathrm{~m} \AA$ & $\cdots$ \\
\hline 46 & HIP 44713 & $\mathrm{m \AA}$ & $\ldots$ \\
\hline 47 & HIP 44935 & $\mathrm{~m} \AA$ & $\ldots$ \\
\hline 48 & HIP 44997 & $\mathrm{mÅ}$ & $\cdots$ \\
\hline 49 & HIP 4909 & $\mathrm{mÅ}$ & $\cdots$ \\
\hline 50 & HIP 49756 & $\mathrm{~mA}$ & $\ldots$ \\
\hline 51 & HIP 5301 & $\mathrm{~m} \AA$ & $\ldots$ \\
\hline 52 & HIP 54102 & $\mathrm{m \AA}$ & $\ldots$ \\
\hline 53 & HIP 54287 & $\mathrm{~m} \AA$ & $\ldots$ \\
\hline 54 & HIP 54582 & $\mathrm{~m} \AA$ & $\ldots$ \\
\hline 55 & HIP 62039 & $\mathrm{~mA}$ & $\ldots$ \\
\hline 56 & HIP 6407 & $\mathrm{mÅ}$ & $\ldots$ \\
\hline 57 & HIP 64150 & $\mathrm{~m} \AA$ & $\ldots$ \\
\hline 58 & HIP 64673 & $\mathrm{m \AA}$ & $\ldots$ \\
\hline 59 & HIP 64713 & $\mathrm{~m} \AA$ & $\ldots$ \\
\hline 60 & HIP 65708 & $\mathrm{mÅ}$ & $\cdots$ \\
\hline 61 & HIP 68468 & $\mathrm{m \AA}$ & $\ldots$ \\
\hline 62 & HIP 69645 & $\mathrm{~mA}$ & $\ldots$ \\
\hline 63 & HIP 72043 & $\mathrm{~mA}$ & $\ldots$ \\
\hline 64 & HIP 73241 & $\mathrm{~m} \AA$ & $\ldots$ \\
\hline
\end{tabular}

Table 1

(Continued)

\begin{tabular}{|c|c|c|c|}
\hline Num & Name & Units & Notes \\
\hline 65 & HIP 73815 & $\mathrm{~m} \AA$ & $\ldots$ \\
\hline 66 & HIP 74389 & $\mathrm{~m} \AA$ & $\ldots$ \\
\hline 67 & HIP 74432 & $\mathrm{~m} \AA$ & $\ldots$ \\
\hline 68 & HIP 7585 & $m \AA ̊$ & $\ldots$ \\
\hline 69 & HIP 76114 & $\mathrm{~m} \AA$ & $\ldots$ \\
\hline 70 & HIP 77052 & $\mathrm{m \AA}$ & ... \\
\hline 71 & HIP 77883 & $\mathrm{m \AA}$ & $\ldots$ \\
\hline 72 & HIP 79578 & $\mathrm{~m} \AA$ & $\ldots$ \\
\hline 73 & HIP 79672 & $\mathrm{m \AA}$ & ... \\
\hline 74 & HIP 79715 & $\mathrm{~m} \AA$ & $\ldots$ \\
\hline 75 & HIP 81746 & $\mathrm{~m} \AA$ & $\ldots$ \\
\hline 76 & HIP 83276 & $\mathrm{~m} \AA$ & $\ldots$ \\
\hline 77 & HIP 85042 & $\mathrm{~m} \AA$ & $\ldots$ \\
\hline 78 & HIP 8507 & $\mathrm{~m} \AA$ & $\ldots$ \\
\hline 79 & HIP 87769 & $\mathrm{~m} \AA$ & $\ldots$ \\
\hline 80 & HIP 89650 & $\mathrm{~m} \AA$ & $\ldots$ \\
\hline 81 & HIP 9349 & $\mathrm{m \AA}$ & $\ldots$ \\
\hline 82 & HIP 95962 & $\mathrm{~m} \AA$ & ... \\
\hline 83 & HIP 96160 & $\mathrm{~m} \AA$ & ... \\
\hline 84 & Sun & $\mathrm{m} \AA$ & $\ldots$ \\
\hline
\end{tabular}

(This table is available in its entirety in machine-readable form.)

Nissen (2015), and Ba, where Spina et al.'s (2018) abundances have an offset of -0.02 dex relative to Nissen (2016). In the case of oxygen, this discrepancy may be due to the use of different lines and slightly different stellar parameters. For barium, the line lists of the two works are nearly identical, but small differences in the measured equivalent widths and the microturbulent velocity parameters adopted may play a significant role for these strong lines.

\subsection{Stellar Ages}

We obtained age estimates for all stars using fits to YonseiYale isochrones (Demarque et al. 2004). A joint fit was performed using our measured $T_{\text {eff }}, \log g$, and $[\mathrm{Fe} / \mathrm{H}]$ as well as the absolute $V$ magnitude inferred from parallaxes. Gaia DR1 photometry and parallaxes were used when available (Gaia Collaboration et al. 2016); data for the other stars came from the All-Sky Compiled Catalog and Tycho-2 (Høg et al. 2000; Kharchenko 2001). We additionally applied metallicity corrections from the $[\alpha / \mathrm{H}]$ abundances using $\mathrm{Mg}$ I as a proxy for $\alpha$. For more detail and a full analysis of the ages obtained, we refer to Spina et al. (2018).

\subsection{Galactic Chemical Evolution}

To probe the effects of GCE on the sample abundances, we investigate the dependence of $[\mathrm{X} / \mathrm{Fe}]$ on stellar age.

As done in Nissen (2015) and Spina et al. (2016a), we fit trends between abundance and age with a linear model; however, not all stars are well-fit by this model. We chose to exclude 10 stars with ages above $8 \mathrm{Gyr}$ and a visible enhancement in $\alpha$ elements. These apparently belong to a different population, perhaps originating in the thick disk. The excluded stars are HIP 19911, HIP 14501, HIP 28066, HIP 30476, HIP 33094, HIP 65708, HIP 73241, HIP 74432, HIP 108158, HIP 109821, and HIP 115577. One additional star, HIP 64150, was excluded because its abundances of 
Table 2

Contents of Abundances Table

\begin{tabular}{|c|c|c|c|}
\hline Num & Name & Units & Notes \\
\hline 1 & Star & & Star identifier \\
\hline 2 & {$[\mathrm{C} \mathrm{I} / \mathrm{H}]$} & $\operatorname{dex}$ & \\
\hline 3 & $\mathrm{u} \_[\mathrm{C} \mathrm{I} / \mathrm{H}]$ & dex & Estimated uncertainty \\
\hline 4 & {$[\mathrm{CH} / \mathrm{H}]$} & dex & \\
\hline 5 & $\mathrm{u} \_[\mathrm{CH} / \mathrm{H}]$ & dex & Estimated uncertainty \\
\hline 6 & {$[\mathrm{O} \mathrm{I} / \mathrm{H}]$} & $\operatorname{dex}$ & \\
\hline 7 & u_[O I/H] & dex & Estimated uncertainty \\
\hline 8 & {$[\mathrm{Na} \mathrm{I} / \mathrm{H}]$} & dex & \\
\hline 9 & $\mathrm{u} \_[\mathrm{Na} \mathrm{I} / \mathrm{H}]$ & dex & Estimated uncertainty \\
\hline 10 & {$[\mathrm{Mg} \mathrm{I} / \mathrm{H}]$} & dex & \\
\hline 11 & $\mathrm{u}_{-}[\mathrm{Mg} \mathrm{I} / \mathrm{H}]$ & dex & Estimated uncertainty \\
\hline 12 & {$[\mathrm{Al} \mathrm{I} / \mathrm{H}]$} & $\operatorname{dex}$ & \\
\hline 13 & $\mathrm{u}_{-}[\mathrm{Al} \mathrm{I} / \mathrm{H}]$ & dex & Estimated uncertainty \\
\hline 14 & {$[\mathrm{Si} \mathrm{I} / \mathrm{H}]$} & dex & \\
\hline 15 & $\mathrm{u}_{-}[\mathrm{Si} \mathrm{I} / \mathrm{H}]$ & dex & Estimated uncertainty \\
\hline 16 & {$[\mathrm{~S} \mathrm{I} / \mathrm{H}]$} & dex & \\
\hline 17 & $\mathrm{u} \_[\mathrm{S} \mathrm{I} / \mathrm{H}]$ & $\operatorname{dex}$ & Estimated uncertainty \\
\hline 18 & {$[\mathrm{Ca} \mathrm{I} / \mathrm{H}]$} & $\operatorname{dex}$ & \\
\hline 19 & $\mathrm{u}_{-}[\mathrm{Ca} \mathrm{I} / \mathrm{H}]$ & dex & Estimated uncertainty \\
\hline 20 & {$[\mathrm{Sc} \mathrm{I} / \mathrm{H}]$} & dex & \\
\hline 21 & $\mathrm{u}_{-}[\mathrm{Sc} \mathrm{I} / \mathrm{H}]$ & dex & Estimated uncertainty \\
\hline 22 & {$[\mathrm{Sc} \mathrm{II} / \mathrm{H}]$} & $\operatorname{dex}$ & \\
\hline 23 & $\mathrm{u} \_[\mathrm{Sc} \text { II } / \mathrm{H}]$ & $\operatorname{dex}$ & Estimated uncertainty \\
\hline 24 & {$[\mathrm{Ti} \mathrm{I} / \mathrm{H}]$} & $\operatorname{dex}$ & \\
\hline 25 & u_[Ti I/H] & dex & Estimated uncertainty \\
\hline 26 & {$[\mathrm{Ti} \mathrm{II} / \mathrm{H}]$} & dex & \\
\hline 27 & $\mathrm{u} \_[\mathrm{Ti} \mathrm{II} / \mathrm{H}]$ & dex & Estimated uncertainty \\
\hline 28 & {$[\mathrm{~V} \mathrm{I} / \mathrm{H}]$} & dex & \\
\hline 29 & $\mathrm{u} \_[\mathrm{V} \mathrm{I} / \mathrm{H}]$ & dex & Estimated uncertainty \\
\hline 30 & {$[\mathrm{Cr} \mathrm{I} / \mathrm{H}]$} & dex & \\
\hline 31 & $\mathrm{u}_{-}[\mathrm{Cr} \mathrm{I} / \mathrm{H}]$ & dex & Estimated uncertainty \\
\hline 32 & {$[\mathrm{Cr}$ II $/ \mathrm{H}]$} & dex & \\
\hline 33 & $\mathrm{u}_{-}[\mathrm{Cr}$ II $/ \mathrm{H}]$ & dex & Estimated uncertainty \\
\hline 34 & {$[\mathrm{Mn} \mathrm{I} / \mathrm{H}]$} & dex & \\
\hline 35 & $\mathrm{u} \_[\mathrm{Mn} \mathrm{I} / \mathrm{H}]$ & $\operatorname{dex}$ & Estimated uncertainty \\
\hline 36 & {$[\mathrm{Co} \mathrm{I} / \mathrm{H}]$} & $\operatorname{dex}$ & \\
\hline 37 & $\mathrm{u}_{-}[\mathrm{Co} \mathrm{I} / \mathrm{H}]$ & dex & Estimated uncertainty \\
\hline 38 & {$[\mathrm{Ni} \mathrm{I} / \mathrm{H}]$} & dex & \\
\hline 39 & $\mathrm{u}_{-}[\mathrm{Ni} \mathrm{I} / \mathrm{H}]$ & dex & Estimated uncertainty \\
\hline 40 & {$[\mathrm{Cu} \mathrm{I} / \mathrm{H}]$} & dex & \\
\hline 41 & $\mathrm{u} \_[\mathrm{Cu} \mathrm{I} / \mathrm{H}]$ & $\operatorname{dex}$ & Estimated uncertainty \\
\hline 42 & {$[\mathrm{Zn} \mathrm{I} / \mathrm{H}]$} & $\operatorname{dex}$ & \\
\hline 43 & $\mathrm{u} \_[\mathrm{Zn} \mathrm{I} / \mathrm{H}]$ & dex & Estimated uncertainty \\
\hline
\end{tabular}

(This table is available in its entirety in machine-readable form.)

several $s$-process elements are highly anomalous, potentially as a result of past accretion from its close binary companion (dos Santos et al. 2017; Spina et al. 2018).

The linear models were fit using an objective function incorporating non-negligible measurement uncertainties in both $(x, y)$ variables (Hogg et al. 2010). In brief, we minimize the orthogonal distances between the linear model and each data point weighted by the data uncertainties. We assume no covariance in these uncertainties. We additionally incorporate a "jitter" or white-noise term added in quadrature with the measurement uncertainties, accounting for the intrinsic star-tostar scatter in abundances. The resulting model has three free parameters for each element: abundance-age slope $m$, intercept $b$, and intrinsic scatter $s$.

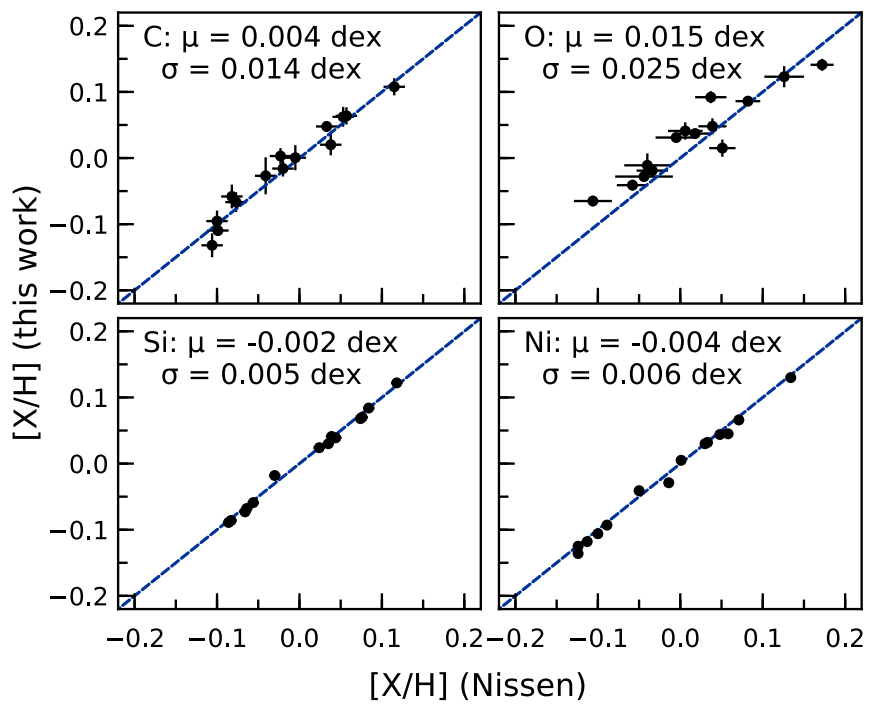

Figure 2. Comparison of abundances derived in this work with those from Nissen (2015) for the 14 overlapping stars in the sample. The mean and standard deviation of the differences are given in each panel.

We find best-fit values using the BFGS algorithm as implemented in the scipy.optimize.minimize module, and uncertainties from an MCMC using emcee (ForemanMackey et al. 2013) with flat priors on all parameters.

For four elements $(\mathrm{C}, \mathrm{Ti}, \mathrm{Sc}$, and $\mathrm{Cr})$, abundances were measured using multiple ionization or molecular states. In principle, the linear fits should be identical across states. In reality, though, the limited line lists used and systematics-prone nature of spectroscopic analyses mean that different degrees of scatter and even systematic offsets could be present between multiple species of the same element. To account for this possibility, we perform joint fits on the two species with slope $m$ required to be the same and $b, s$ allowed to be different for each one. The resulting best-fit GCE trends are shown in Figure 3. Best-fit parameters and uncertainties are given in Table 3.

We note that none of the elements that were measured in multiple species display a systematic offset in their $b$ parameters beyond the level expected from random scatter. The intrinsic scatter parameter $s$ tends to be largest for those species for which few lines were measured, including $\mathrm{Na}$ I (4 lines), Cu I (3 lines), O I (3 lines), and the carbon species. This is likely a reflection of the statistical tendency to underestimate uncertainties in the limit of small samples (Adibekyan et al. 2015a). For species with a more extensive list of easy-to-measure lines, like Ti I and Ni I (18 lines each), the intrinsic scatter remains significantly nonzero. This may be a reflection of the true degree of inherent deviations in individual stars from the GCE-predicted average abundance pattern.

\subsection{Condensation Temperature Trends}

The calculation of trends in elemental abundance with $T_{\mathrm{C}}$ is an especially sensitive measurement that can be strongly affected by small inaccuracies in stellar parameters or in the abundances of key volatile elements (e.g., Adibekyan et al. 2016; Teske et al. 2016). Moreover, it is not immediately obvious what model should be used to fit this trend. In our analysis, we take a few precautions to maximize the accuracy with which we detect refractory element depletions or enhancements in the sample. 


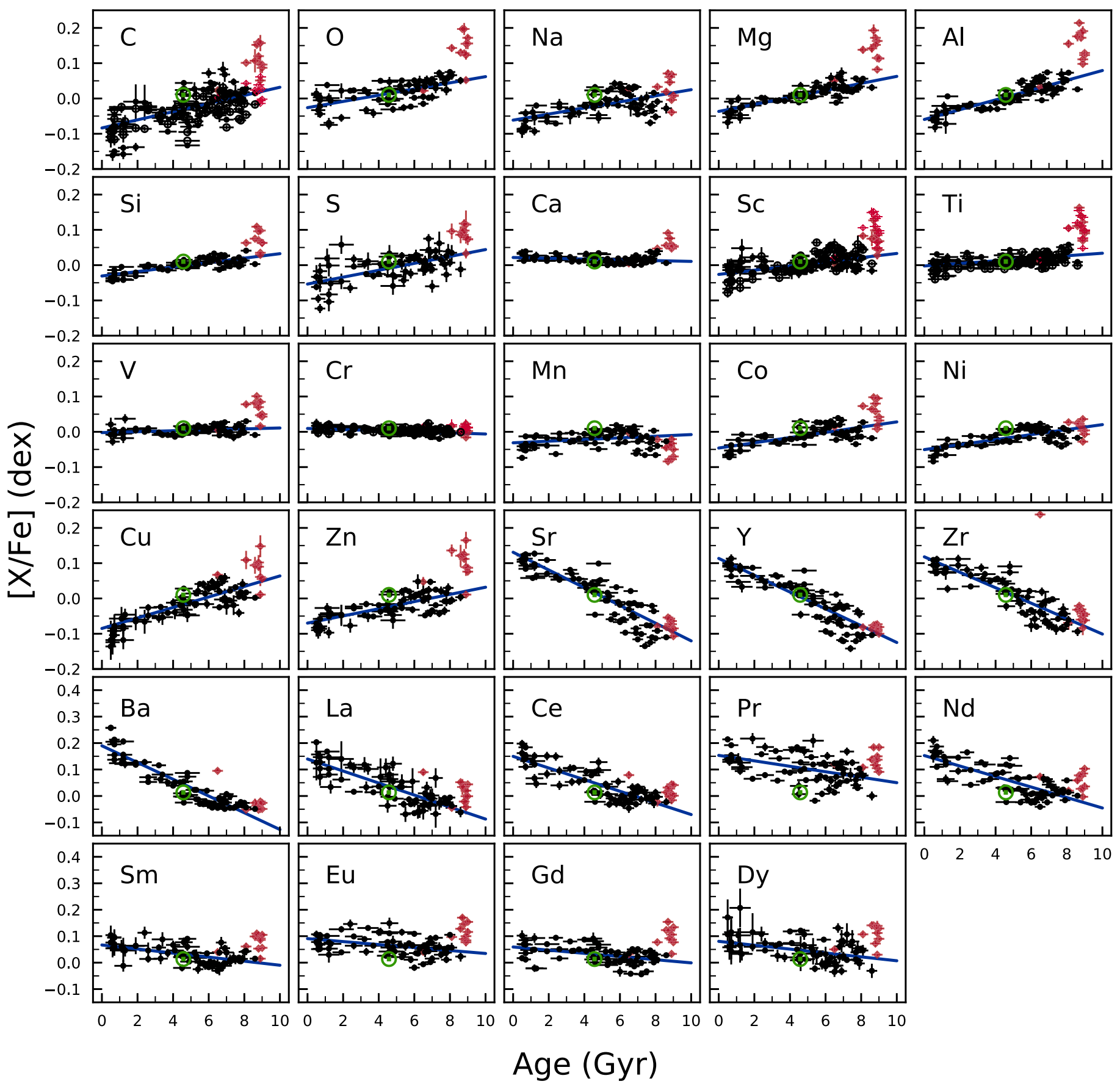

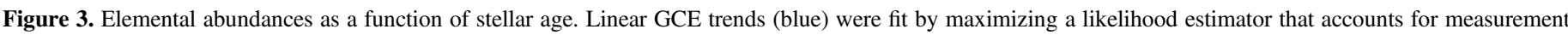

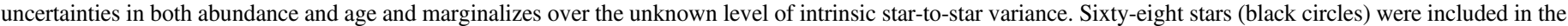

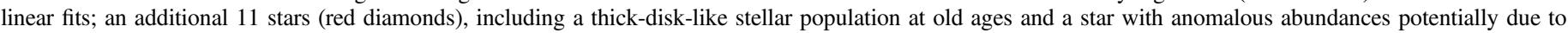

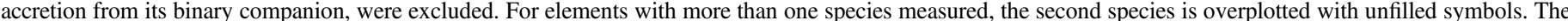
solar abundances (green) were not considered in the fit, but are nevertheless consistent with it.

When fitting a linear trend to the abundances, we exclude the volatile elements $\mathrm{C}, \mathrm{O}, \mathrm{S}$, and $\mathrm{Zn}$. Based on the $T_{\mathrm{C}}$ trends seen in solar-system meteorites, these elements are not expected to fall along the steepest portion of the refractory-driven $T_{\mathrm{C}}$ slope (Chambers 2010, and references therein). Meléndez et al. (2009) accounted for the varying $T_{\mathrm{C}}$ behavior of the volatile and refractory elements by fitting with a piecewise linear function, but the location of the line break is not welldetermined. Although some subsequent works have included the volatiles in a simple linear fit with the other elements, this approach has its own complications. As noted by Adibekyan et al. (2016), elements with under- or overestimated abundance uncertainties are prone to biasing the overall $T_{\mathrm{C}}$ trend if included in the fit. This can especially lead to bias in the case of the most volatile elements $\left(T_{\mathrm{C}} \lesssim 900 \mathrm{~K}\right)$, as a higher proportion of these elements have uncertain errors due to the small numbers of lines available and the potential systematic effects due to imperfect NLTE corrections. In addition, volatiles like $\mathrm{C}$ and $\mathrm{O}$ may condense under a range of conditions other than their nominal $T_{\mathrm{C}}$ values due to their incorporation in a wide 
Table 3

Best-fit Parameters for GCE Fits

\begin{tabular}{|c|c|c|c|}
\hline Species & $m\left(\operatorname{dex} \mathrm{Gyr}^{-1}\right)$ & $b(\mathrm{dex})$ & $s(\mathrm{dex})$ \\
\hline C I & $0.0115 \pm 0.0014$ & $-0.0836 \pm 0.0089$ & $0.0394 \pm 0.0044$ \\
\hline $\mathrm{CH}$ & $0.0115 \pm 0.0014$ & $-0.0940 \pm 0.0079$ & $0.0293 \pm 0.0030$ \\
\hline O I & $0.0088 \pm 0.0014$ & $-0.0260 \pm 0.0075$ & $0.0238 \pm 0.0028$ \\
\hline $\mathrm{Na} I$ & $0.0086 \pm 0.0016$ & $-0.0614 \pm 0.0089$ & $0.0273 \pm 0.0029$ \\
\hline Mg I & $0.0099 \pm 0.0009$ & $-0.0367 \pm 0.0048$ & $0.0121 \pm 0.0018$ \\
\hline $\mathrm{Al} \mathrm{I}$ & $0.0139 \pm 0.0010$ & $-0.0595 \pm 0.0054$ & $0.0156 \pm 0.0019$ \\
\hline Si I & $0.0063 \pm 0.0006$ & $-0.0308 \pm 0.0033$ & $0.0110 \pm 0.0011$ \\
\hline S I & $0.0098 \pm 0.0015$ & $-0.0537 \pm 0.0085$ & $0.0240 \pm 0.0035$ \\
\hline $\mathrm{Ca} \mathrm{I}$ & $-0.0011 \pm 0.0006$ & $0.0217 \pm 0.0032$ & $0.0089 \pm 0.0011$ \\
\hline Sc I & $0.0059 \pm 0.0009$ & $-0.0263 \pm 0.0052$ & $0.0159 \pm 0.0022$ \\
\hline Sc II & $0.0059 \pm 0.0009$ & $-0.0235 \pm 0.0052$ & $0.0205 \pm 0.0025$ \\
\hline Ti I & $0.0036 \pm 0.0005$ & $-0.0024 \pm 0.0032$ & $0.0119 \pm 0.0014$ \\
\hline Ti II & $0.0036 \pm 0.0005$ & $-0.0094 \pm 0.0031$ & $0.0115 \pm 0.0014$ \\
\hline V I & $0.0013 \pm 0.0007$ & $-0.0023 \pm 0.0037$ & $0.0091 \pm 0.0011$ \\
\hline Cr I & $-0.0016 \pm 0.0003$ & $0.0095 \pm 0.0019$ & $0.0053 \pm 0.0008$ \\
\hline Cr II & $-0.0016 \pm 0.0003$ & $0.0133 \pm 0.0019$ & $0.0000 \pm 0.0001$ \\
\hline Mn I & $0.0023 \pm 0.0012$ & $-0.0312 \pm 0.0063$ & $0.0206 \pm 0.0020$ \\
\hline Co I & $0.0074 \pm 0.0011$ & $-0.0460 \pm 0.0057$ & $0.0178 \pm 0.0020$ \\
\hline Ni I & $0.0071 \pm 0.0009$ & $-0.0505 \pm 0.0050$ & $0.0172 \pm 0.0018$ \\
\hline $\mathrm{Cu} \mathrm{I}$ & $0.0149 \pm 0.0017$ & $-0.0850 \pm 0.0097$ & $0.0244 \pm 0.0030$ \\
\hline $\mathrm{Zn} \mathrm{I}$ & $0.0102 \pm 0.0014$ & $-0.0699 \pm 0.0077$ & $0.0224 \pm 0.0029$ \\
\hline Sr I & $-0.0251 \pm 0.0030$ & $0.1310 \pm 0.0164$ & $0.0574 \pm 0.0060$ \\
\hline Y II & $-0.0238 \pm 0.0024$ & $0.1135 \pm 0.0130$ & $0.0470 \pm 0.0051$ \\
\hline Zr II & $-0.0219 \pm 0.0023$ & $0.1179 \pm 0.0125$ & $0.0422 \pm 0.0046$ \\
\hline Ba II & $-0.0317 \pm 0.0018$ & $0.1897 \pm 0.0093$ & $0.0309 \pm 0.0039$ \\
\hline La II & $-0.0227 \pm 0.0021$ & $0.1397 \pm 0.0121$ & $0.0350 \pm 0.0046$ \\
\hline $\mathrm{Ce}$ II & $-0.0220 \pm 0.0018$ & $0.1497 \pm 0.0097$ & $0.0305 \pm 0.0039$ \\
\hline Pr II & $-0.0103 \pm 0.0025$ & $0.1534 \pm 0.0131$ & $0.0451 \pm 0.0050$ \\
\hline Nd II & $-0.0198 \pm 0.0020$ & $0.1527 \pm 0.0108$ & $0.0360 \pm 0.0038$ \\
\hline Sm II & $-0.0077 \pm 0.0017$ & $0.0668 \pm 0.0094$ & $0.0226 \pm 0.0032$ \\
\hline Eu II & $-0.0056 \pm 0.0017$ & $0.0908 \pm 0.0093$ & $0.0300 \pm 0.0033$ \\
\hline Gd II & $-0.0060 \pm 0.0016$ & $0.0592 \pm 0.0089$ & $0.0279 \pm 0.0028$ \\
\hline Dy II & $-0.0073 \pm 0.0023$ & $0.0805 \pm 0.0123$ & $0.0332 \pm 0.0043$ \\
\hline
\end{tabular}

variety of compounds, adding a further layer of complexity to the interpretation of these elements from the standpoint of dust condensation processes (Lodders 2003). For these reasons, we performed our primary $T_{\mathrm{C}}$ fits using a single linear model and considering only the 26 elements with $T_{\mathrm{C}}>900 \mathrm{~K}$.

It has previously been noted that GCE may affect the $T_{\mathrm{C}}$ trends of stars at different ages (Adibekyan et al. 2014). We make use of our constraints on the abundance-age relations in the sample to subtract off inferred GCE effects from each star's abundances. The GCE-corrected abundances were calculated using the previously derived best-fit GCE trends, with the estimated errors on the GCE slopes propagated to the abundance uncertainties by adding their contributions in quadrature with the existing line-scatter-based uncertainty estimates. The 11 stars that were excluded from the GCE fits were dropped from the post-GCE-correction sample.

We calculated $T_{\mathrm{C}}$ trends for every star in the sample by fitting the $[\mathrm{X} / \mathrm{H}]$ abundances and uncertainties with a nonlinear leastsquares optimizer as implemented in scipy.optimize. least_squares. The $T_{\mathrm{C}}$ values were taken as $50 \%$ condensation temperatures based on calculations for solar-system composition gas (Lodders 2003). For each star, a $T_{\mathrm{C}}$ slope was calculated both from the "raw" $[\mathrm{X} / \mathrm{H}]$ abundances and also from the abundances after subtracting GCE effects.

To verify the robustness of our results, we also consider a piecewise linear function as an alternative model. We performed fits in this case including all elements and allowing the break point of the piecewise function to vary as a free parameter. We adopt the slope of the high- $T_{\mathrm{C}}$ component as the nominal $T_{\mathrm{C}}$ slope for these fits.

The resulting $T_{\mathrm{C}}$ slopes for each star are presented in Table 4. We plot a few representative stars in Figure 4 to illustrate the range of behaviors observed.

\subsection{The Average Solar Twin}

Finally, following Meléndez et al. (2009), we calculate the abundances of a "typical" solar twin and compare this abundance pattern to that of the Sun. We calculate the sample average in linear space with the number density of atoms; that is, we take the detailed abundance pattern of the average star to be composed of an equal mixture of materials from the photosphere of every twin. The average abundance ratio of element $X$ to element $Y$ across the full sample of $\mathrm{N}$ stars is therefore defined as:

$$
\left\langle\left[\frac{X}{Y}\right]\right\rangle=\log _{10}\left(\frac{1}{N} \sum_{n=0}^{N} 10^{\left[\frac{X}{Y}\right]_{n}}\right) .
$$

In Section 3.4, we used $[\mathrm{X} / \mathrm{H}]$ rather than $[\mathrm{X} / \mathrm{Fe}]$ to calculate $T_{\mathrm{C}}$ trends, as recommended by Adibekyan et al. (2016). For an individual star with well-determined $[\mathrm{Fe} / \mathrm{H}]$, either abundance ratio will yield virtually the same $T_{\mathrm{C}}$ slope with only the offset parameter changing, and by adopting $[\mathrm{X} / \mathrm{H}]$ we are able to include $[\mathrm{Fe} / \mathrm{H}]$ as an additional data point. When determining the sample average abundances, though, the propagation of individual stars' $[\mathrm{Fe} / \mathrm{H}]$ makes a difference to the average calculated abundances (and therefore the resulting $T_{\mathrm{C}}$ slope). We made the choice to use $[\mathrm{X} / \mathrm{Fe}]$ in an attempt to minimize the potential effects of diffusion processes in stars of different ages or slightly different masses. The magnitude of diffusion effects should be much smaller when comparing an elemental abundance to iron (which diffuses out of the photosphere like the other measured elements) than when comparing to hydrogen (which actually increases in photospheric concentration over time). While some residual effects of diffusion will be present in the ratios of elements with varying masses and ionic charges, it is expected that normalizing for $[\mathrm{Fe} / \mathrm{H}]$ will reduce these effects to the level of the measurement uncertainties (Dotter et al. 2017).

In practice, the difference between $\left\langle\left[\frac{X}{F e}\right]\right\rangle$ and $\left\langle\left[\frac{x}{H}\right]\right\rangle$ is negligible (below 0.005 dex for all elements), and we find the same average $T_{\mathrm{C}}$ trend regardless. The trend was fit as in Section 3.4, with abundance uncertainties from the scatter among the sample. The resulting $T_{\mathrm{C}}$ fits for the sample and for the GCE-corrected sample are shown in Figure 5.

\section{Results and Discussion}

\subsection{Galactic Chemical Evolution}

The observed GCE trends among our sample (Figure 3) generally follow the trends of expected behavior from nucleosynthetic theory. The $\alpha$-elements like magnesium and silicon increase with age as the occurrence of type Ia supernovae relative to type II is lower in the earlier universe, with a discontinuity seen in the oldest, thick-disk-like stellar populations (Gilmore et al. 1989). Sodium, as an odd- $Z$ light element, follows a similar trend but shows hints of more complex evolution, as has been previously noted in studies of sodium abundance as a function of metallicity (Bensby et al. 2017). On the other hand, the iron-peak element 
Table 4

$T_{\mathrm{C}}$ Fits for the Sample

\begin{tabular}{|c|c|c|c|c|}
\hline Star & $\begin{array}{c}\text { Slope }^{\mathrm{a}} \\
\left(10^{-4} \mathrm{dex} \mathrm{K}^{-1}\right)\end{array}$ & $\begin{array}{l}\text { GCE-corrected Slope }{ }^{\mathrm{a}} \\
\left(10^{-4} \mathrm{dex} \mathrm{K}^{-1}\right)\end{array}$ & $\begin{array}{c}\text { Slope } \\
\left(10^{-4} \text { dex K }^{-1}\right)\end{array}$ & $\begin{array}{c}\text { GCE-corrected Slope } \\
\left(10^{-4} \text { dex K }^{-1}\right)\end{array}$ \\
\hline HIP 10175 & 2.35 & 1.90 & 2.39 & 1.96 \\
\hline HIP 101905 & 0.48 & 1.39 & 0.58 & 1.39 \\
\hline HIP 102040 & 2.52 & 1.91 & 2.71 & 2.03 \\
\hline HIP 102152 & -0.04 & 0.89 & -0.04 & 0.89 \\
\hline HIP 10303 & 0.12 & 0.34 & 0.17 & 0.33 \\
\hline HIP 104045 & 0.36 & 0.39 & 0.36 & 0.39 \\
\hline HIP 105184 & 3.01 & 1.75 & 3.15 & 1.90 \\
\hline HIP 108158 & 0.07 & 1.17 & 0.04 & 1.34 \\
\hline HIP 108468 & 0.49 & 1.25 & 0.49 & 1.25 \\
\hline HIP 109821 & 1.66 & 2.49 & 1.86 & 2.59 \\
\hline HIP 114328 & -0.26 & -0.10 & 1.32 & -0.01 \\
\hline HIP 114615 & 2.99 & 1.05 & 3.13 & 1.13 \\
\hline HIP 115577 & -0.03 & 1.51 & -0.26 & 1.50 \\
\hline HIP 116906 & -0.56 & 0.43 & -0.58 & 0.43 \\
\hline HIP 117367 & -0.51 & -0.19 & -0.49 & -0.17 \\
\hline HIP 118115 & 0.20 & 1.60 & 0.20 & 1.60 \\
\hline HIP 11915 & 0.38 & 1.10 & 0.35 & 1.12 \\
\hline HIP 14501 & 0.86 & 2.14 & 2.43 & 2.38 \\
\hline HIP 14614 & 1.50 & 1.62 & 1.58 & 1.74 \\
\hline HIP 15527 & 0.20 & 1.19 & 0.20 & 1.19 \\
\hline HIP 18844 & 0.06 & 0.14 & 0.13 & 0.17 \\
\hline HIP 1954 & 1.31 & 1.25 & 1.66 & 1.40 \\
\hline HIP 22263 & 3.17 & 1.58 & 3.35 & 1.66 \\
\hline HIP 25670 & 0.46 & 0.60 & 0.46 & 0.61 \\
\hline HIP 28066 & 1.76 & 2.33 & 1.42 & 2.56 \\
\hline HIP 29432 & 0.76 & 0.97 & 0.82 & 1.08 \\
\hline HIP 29525 & 3.70 & 1.84 & 4.57 & 2.11 \\
\hline HIP 30037 & 0.24 & 0.75 & 0.20 & 0.74 \\
\hline HIP 30158 & 0.64 & 0.53 & 0.80 & 0.59 \\
\hline HIP 30476 & 0.43 & 1.61 & 0.45 & 1.83 \\
\hline HIP 30502 & 0.25 & 1.31 & 0.28 & 1.31 \\
\hline HIP 3203 & 2.66 & 2.00 & 2.70 & 2.07 \\
\hline HIP 33094 & -0.08 & 1.23 & 0.19 & 1.66 \\
\hline HIP 34511 & 1.09 & 1.12 & 1.13 & 1.19 \\
\hline HIP 36512 & 0.90 & 1.09 & 1.03 & 1.23 \\
\hline HIP 36515 & 1.77 & 1.58 & 1.77 & 1.57 \\
\hline HIP 38072 & 0.72 & 0.22 & 0.78 & 0.27 \\
\hline HIP 40133 & 0.14 & 0.23 & 0.09 & -0.17 \\
\hline HIP 41317 & 0.35 & 1.44 & 0.35 & 1.44 \\
\hline HIP 42333 & 1.16 & 0.79 & 1.19 & 0.83 \\
\hline HIP 43297 & 1.66 & 1.14 & 1.68 & 1.15 \\
\hline HIP 44713 & -0.39 & 0.84 & -0.34 & 0.76 \\
\hline HIP 44935 & 0.14 & 0.32 & 0.13 & 0.32 \\
\hline HIP 44997 & 0.27 & 0.77 & 0.36 & 0.78 \\
\hline HIP 4909 & 3.53 & 1.75 & 3.71 & 1.85 \\
\hline HIP 49756 & 0.24 & 0.23 & 0.30 & 0.29 \\
\hline HIP 5301 & 0.11 & 1.18 & 0.12 & 1.23 \\
\hline HIP 54102 & 2.22 & 1.65 & 2.24 & 1.68 \\
\hline HIP 54287 & -0.41 & 0.13 & -0.66 & -0.14 \\
\hline HIP 54582 & 0.67 & 1.41 & 0.67 & 1.41 \\
\hline HIP 62039 & -0.60 & -0.08 & -0.64 & -0.10 \\
\hline HIP 6407 & 1.49 & 1.31 & 1.51 & 1.41 \\
\hline HIP 64150 & 1.73 & 2.35 & 2.47 & 3.53 \\
\hline HIP 64673 & -0.52 & -0.30 & -0.42 & -1.90 \\
\hline HIP 64713 & 0.77 & 1.03 & 0.80 & 1.08 \\
\hline HIP 65708 & 1.25 & 2.28 & 1.76 & 2.83 \\
\hline HIP 68468 & -0.12 & 0.05 & -0.10 & 0.07 \\
\hline HIP 69645 & -0.05 & 0.09 & 0.06 & 0.16 \\
\hline HIP 72043 & 0.42 & 0.24 & 0.74 & 0.29 \\
\hline HIP 73241 & 1.09 & 1.31 & 1.30 & 1.27 \\
\hline HIP 73815 & 0.10 & 0.28 & 0.11 & 0.28 \\
\hline HIP 74389 & 1.38 & 1.12 & 1.40 & 1.16 \\
\hline HIP 74432 & 0.58 & 2.01 & 0.52 & 2.31 \\
\hline
\end{tabular}


Table 4

(Continued)

\begin{tabular}{|c|c|c|c|c|}
\hline Star & $\begin{array}{c}\text { Slope }^{\mathrm{a}} \\
\left(10^{-4} \text { dex K }^{-1}\right)\end{array}$ & $\begin{array}{l}\text { GCE-corrected Slope }{ }^{\mathrm{a}} \\
\left(10^{-4} \text { dex K }{ }^{-1}\right)\end{array}$ & $\begin{array}{c}\text { Slope } \\
\left(10^{-4} \operatorname{dex~K}^{-1}\right)\end{array}$ & $\begin{array}{l}\text { GCE-corrected Slope } \\
\left(10^{-4} \mathrm{dex} \mathrm{K}^{-1}\right)\end{array}$ \\
\hline HIP 7585 & 1.16 & 0.74 & 1.75 & 1.00 \\
\hline HIP 76114 & -0.23 & 0.45 & -0.19 & 0.49 \\
\hline HIP 77883 & -0.80 & 0.46 & -0.74 & 0.46 \\
\hline HIP 79578 & 0.83 & 0.28 & 0.91 & 0.24 \\
\hline HIP 79715 & -0.51 & 0.05 & -0.51 & 0.07 \\
\hline HIP 83276 & 0.48 & 1.19 & 0.48 & 1.19 \\
\hline HIP 85042 & 0.64 & 1.43 & 0.64 & 1.46 \\
\hline HIP 8507 & 0.66 & 1.42 & 0.66 & 1.42 \\
\hline HIP 87769 & 0.23 & 0.22 & 0.11 & 0.09 \\
\hline HIP 89650 & -0.16 & -0.17 & -0.17 & -0.17 \\
\hline HIP 9349 & 1.02 & 0.63 & 1.02 & 0.68 \\
\hline
\end{tabular}

Notes.

${ }^{\mathrm{a}}$ From linear fit (see Section 3.4 for model details).

${ }^{\mathrm{b}}$ From piecewise linear model fit.

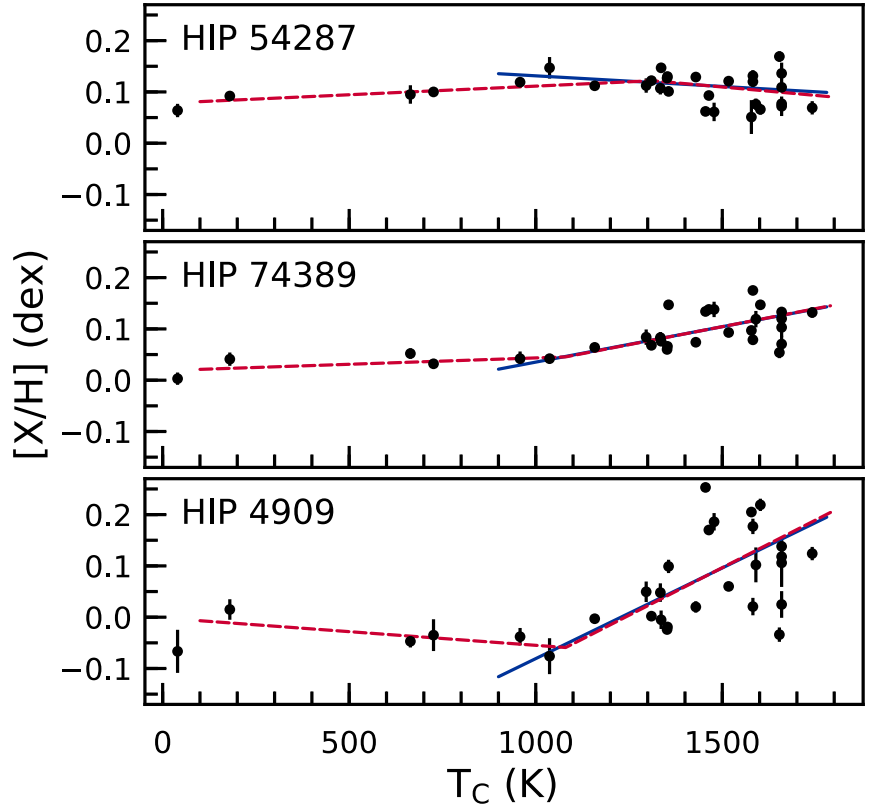

Figure 4. Example stars from the sample demonstrating the range of $T_{\mathrm{C}}$ trend behavior observed. For each star, differential abundances are shown as a function of the elements' expected condensation temperatures $\left(T_{\mathrm{C}}\right)$ in the protoplanetary disk (Lodders 2003). Best-fit linear (solid blue line) and piecewise linear (dashed red line) fits are also shown.

chromium varies identically to iron through time, as expected. Beyond these general categories of nucleosynthetic production, we can resolve more subtle differences among the $[\mathrm{X} / \mathrm{Fe}]$ versus age behaviors for individual elements. Figure 6 aggregates the observed GCE trends as a function of atomic number. In addition to broad-stroke differences like heavy-element $(Z>30)$ abundances decreasing with stellar age and lighter elements increasing, the element-to-element scatter in slope exceeds statistical uncertainties even within a nucleosynthetic group. Among the heavy neutron-capture elements, a correlation with atomic number can be seen; this behavior was previously shown

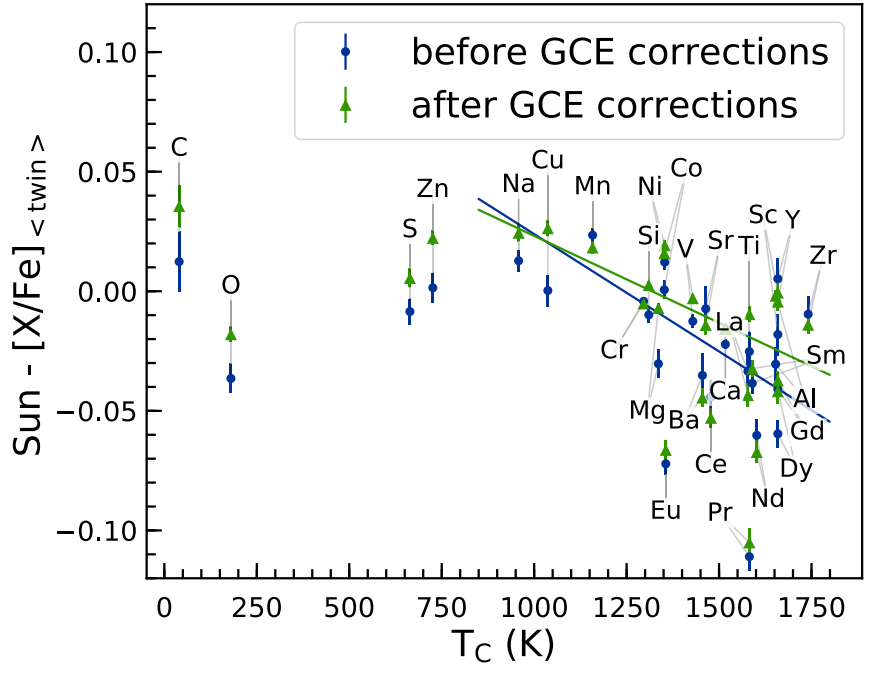

Figure 5. Abundance pattern of the Sun compared to the average values in the solar twin sample. Error bars on the abundances are empirically derived as the $1 \sigma$ error on the mean of the sample. The abundances shown are derived from the full 79-star sample (blue circles) and for the GCE-corrected 68-star subsample (green triangles, see the text for details). Linear trends are fit to 25 refractory elements and the best-fit lines are shown. Relative to the typical solar twin, the Sun appears deficient in refractory materials (or enhanced in volatiles).

in Spina et al. (2018) and explained in terms of the changing fractional contributions of $s$ - and $r$-processes. Among the lighter elements, calcium is a notable outlier, having a slope close to zero despite its nominal status as an alpha-element. This behavior, which was also seen by Nissen (2015), may be due to its production in sites with longer delay times in addition to type II supernovae.

A full analysis of the nucleosynthetic information contained in these results would likely require chemodynamical models (e.g., Kobayashi \& Nakasato 2011), but the few examples discussed here serve as a demonstration of the power of these data to constrain nucleosynthetic yields and GCE models. More broadly speaking, the observed GCE trends place the Sun in 


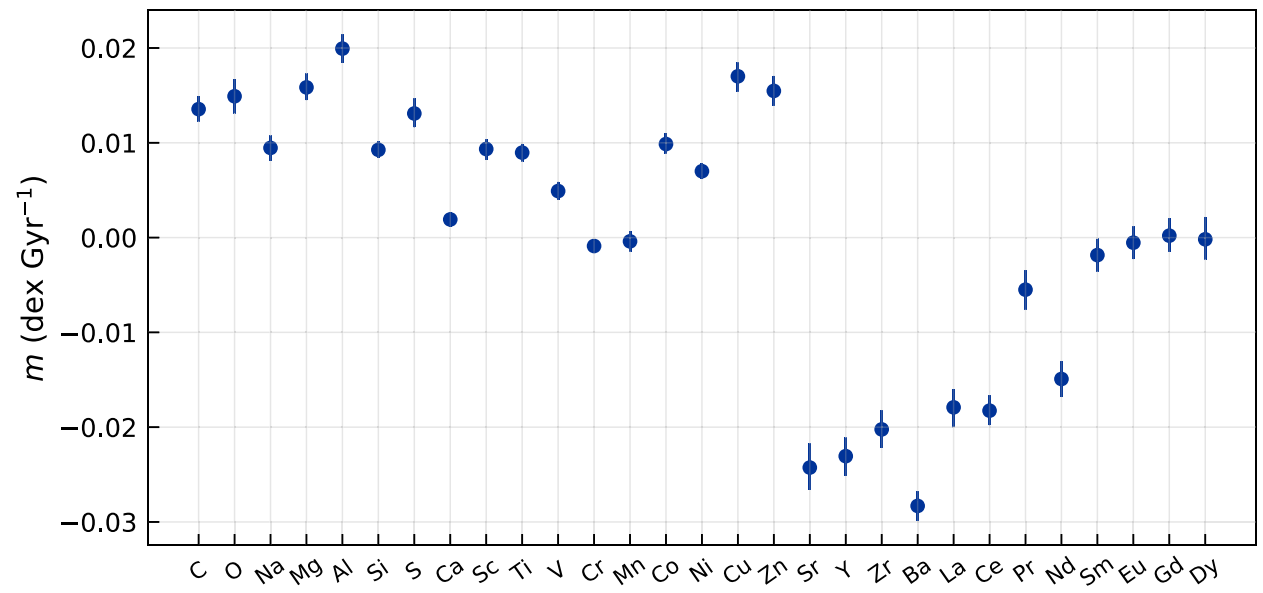

Figure 6. $[\mathrm{X} / \mathrm{Fe}]$ vs. age slopes $m$ for all 29 elements in the sample, ordered by atomic number. The fitting procedure used to derive these parameters is detailed in Section 3.3. Noticeable differences are present between heavy $(Z>30)$ and light elements, with $s$-process dominated elements exhibiting the most extreme age trends, as previously noted by Spina et al. (2018).

context among its neighbors, showing that its chemical composition is characteristic of other solar-metallicity stars formed at the same epoch of galactic history.

\section{2. $\mathrm{C} / \mathrm{O}$ and $\mathrm{Mg} / \mathrm{Si}$ Ratios}

It is well established that the carbon-to-oxygen $(\mathrm{C} / \mathrm{O})$ and magnesium-to-silicon $(\mathrm{Mg} / \mathrm{Si})$ abundance ratios play a key role in the formation, atmospheric chemistry, and interior structure in general for all types of planets, and the possibility of plate tectonics and habitability of terrestrial planets in particular (Kuchner \& Seager 2005; Madhusudhan et al. 2011; Öberg et al. 2011; Unterborn et al. 2014; Unterborn \& Panero 2017). The broad distribution of $\mathrm{C} / \mathrm{O}$ and $\mathrm{Mg} / \mathrm{Si}$ values found in large-scale abundance surveys suggests that a significant fraction of exoplanets are born in environments with very different compositions than that of the primordial solar nebula (Delgado Mena et al. 2010; Petigura \& Marcy 2011; Adibekyan et al. 2012; Bensby et al. 2014; Brewer \& Fischer 2016), and thus could have strikingly different properties than the solar-system planets (Bond et al. 2010; Carter-Bond et al. 2012; Unterborn et al. 2017).

We show the derived $\mathrm{C} / \mathrm{O}$ and $\mathrm{Mg} / \mathrm{Si}$ abundance ratios from our solar twins abundance study in Figure 7. As a comparison sample, FGK stars from the Brewer \& Fischer (2016) sample with $\log g>3.5 \mathrm{dex}$ and $-0.2<[\mathrm{Fe} / \mathrm{H}]<0.2 \mathrm{dex}$ are also shown in the figure. In contrast to previous results, we find very little scatter in these abundance ratios over an $8 \mathrm{Gyr}$ range in age and for iron abundances within \pm 0.15 dex $(40 \%)$ of the solar value. The $\mathrm{C} / \mathrm{O}$ values are fully bounded between 0.4 and 0.6 , while all the $\mathrm{Mg} / \mathrm{Si}$ values are consistent with being greater than unity (compared to the solar values of 0.54 and 1.05, respectively, Asplund et al. 2009). Our results show that these key abundance ratios are quite similar across all Sun-like stars, implying that planetary systems around these stars are born from the same chemical building blocks.

We additionally consider the evolution of these abundance ratios with stellar age and/or metallicity. The $\mathrm{C} / \mathrm{O}$ ratio appears to evolve very little, as predicted by the GCE simulation of Gaidos (2015) and in good agreement with past work by Nissen (2015). Although the variance of $\mathrm{C} / \mathrm{O}$ values is smaller than that found in Brewer \& Fischer (2016), the overall
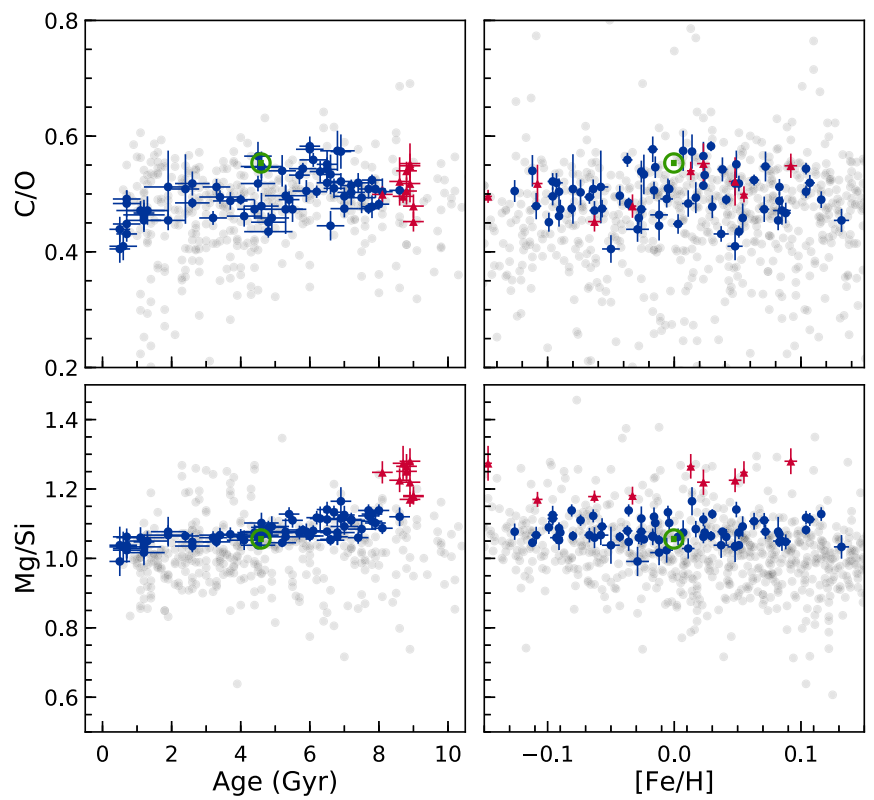

Figure 7. Carbon-to-oxygen (C/O, top row) and magnesium-to-silicon ( $\mathrm{Mg} / \mathrm{Si}$, bottom row) abundance ratios for solar-type stars in the solar neighborhood. Ratios are shown as functions of stellar age (left) and iron abundance (right). Values for a sample of FGK dwarfs in the same metallicity range from Brewer \& Fischer (2016) are plotted in gray for comparison, with estimated uncertainties of $10 \%$ for $\mathrm{C} / \mathrm{O}$ and $3.3 \%$ for $\mathrm{Mg} / \mathrm{Si}$. By reducing model-dependent bias we find that solar twins (plotted in two populations following the same symbol conventions as Figure 3) exhibit lower variance in composition than previously found: in particular, we find no significantly carbon-enriched stars, and all $\mathrm{Mg} / \mathrm{Si}$ values are consistent with being above unity. We also see a clear evolution in $\mathrm{Mg} / \mathrm{Si}$ with age, but no trends with bulk metallicity are observed over the range of values investigated.

placement of the Sun at the high-C/O end of the distribution agrees well.

On the other hand, the $\mathrm{Mg} / \mathrm{Si}$ ratio increases with age. This qualitatively agrees with theory: while both $\mathrm{Mg}$ and $\mathrm{Si}$ are produced by alpha-capture processes in massive stars, there is also non-negligible $\mathrm{Si}$ production from Type Ia supernovae (e.g., Burbidge et al. 1957). In keeping with this ongoing Si production, we observe lower $\mathrm{Mg} / \mathrm{Si}$ in younger stars than in the oldest sample stars. Adibekyan et al. (2015b) found a similar effect when examining the $\mathrm{Mg} / \mathrm{Si}$ ratio for stars across 
a wide range in metallicity, with the lowest $[\mathrm{Fe} / \mathrm{H}]$ population exhibiting the largest $\mathrm{Mg} / \mathrm{Si}$. In this case, we see no significant correlation between $[\mathrm{Fe} / \mathrm{H}]$ and $\mathrm{Mg} / \mathrm{Si}$, which is not surprising over such a small range in metallicity. Age is apparently a more sensitive tracer of the galactic evolution processes that shape the $\mathrm{Mg} / \mathrm{Si}$ ratio within this population.

We can conclude from these results that all nearby planets are formed out of material with Sun-like $\mathrm{C} / \mathrm{O}$ and $\mathrm{Mg} / \mathrm{Si}$ ratios if two additional assumptions hold: that the solar twin abundances are representative of stars of different spectral types, and that $\mathrm{Mg} / \mathrm{Si}$ and $\mathrm{C} / \mathrm{O}$ ratios do not vary appreciably at nonsolar metallicities. Neither of these assumptions is easy to test, as model-based systematics are no longer a negligible contributor to abundances derived for stars of different spectral types and metallicities, or even to a less strictly differential analysis of solar analogs (as in the comparison made in Figure 7). Since the stars in the solar twin sample all have solar metallicity but span a wide range of ages, they must represent a range of nucleosynthetic pathways in galactic star formation, yet all end up with nearly the same $\mathrm{Mg} / \mathrm{Si}$ and $\mathrm{C} / \mathrm{O}$ ratios. This suggests that the results found for solar twins can be more widely extrapolated to stars of other metallicities. Other works, however, have found differences in these ratios as a function of metallicity (Adibekyan et al. 2015b; Santos et al. 2017). In any case, the vast majority of local stars have approximately solar metallicity (e.g., Casagrande et al. 2011), making these compositional constraints likely to apply for most nearby exoplanet hosts.

\subsection{Condensation Temperature Trends}

The results described above suggest that the Sun is entirely typical in its composition. Nevertheless, previous works have debated the existence of one atypical attribute of the solar abundance pattern that may be tied to planet formation: a subtle deficiency in the solar abundances of refractory elements relative to volatiles (Meléndez et al. 2009; Ramírez et al. 2009; Adibekyan et al. 2014; Nissen 2015).

Despite its important repercussions for the search for Earthlike planets, the existence of an unusual $T_{\mathrm{C}}$ trend for the Sun has not been definitively validated due to the difficulty of measuring stellar abundances at the needed level of precision. The amplitude of the proposed solar abundance trend is much smaller than the sensitivity of most traditional abundance surveys, and it is easily overwhelmed by the systematic errors that plague studies of stars with even moderate differences in their physical parameters. Abundance trends may also be obscured by the effects of GCE in a sample of stars with different ages (Adibekyan et al. 2014; Nissen 2015; Spina et al. 2016b). Our results offer an opportunity to investigate the reality of the proposed solar $T_{\mathrm{C}}$ trend for a sample of 79 stars with very precise abundance measurements and well-characterized GCE behavior.

The distribution of $T_{\mathrm{C}}$ trends in our sample is shown in Figure 8. Among our GCE-corrected sample of 68 solar twins, we find only five stars with a $T_{\mathrm{C}}$ slope at or below the level of the Sun, meaning that the Sun shows a refractory-to-volatile deficiency relative to $93 \%$ of the sample. We estimate the error on this result by resampling the slopes with injected noise based on their measured uncertainties and recording the Sun's position within the resulting simulated distribution. In $95 \%$ of the trials, the Sun lies below at least $84 \%$ of solar twins. This result does not appear sensitive to the model used for fitting $T_{\mathrm{C}}$
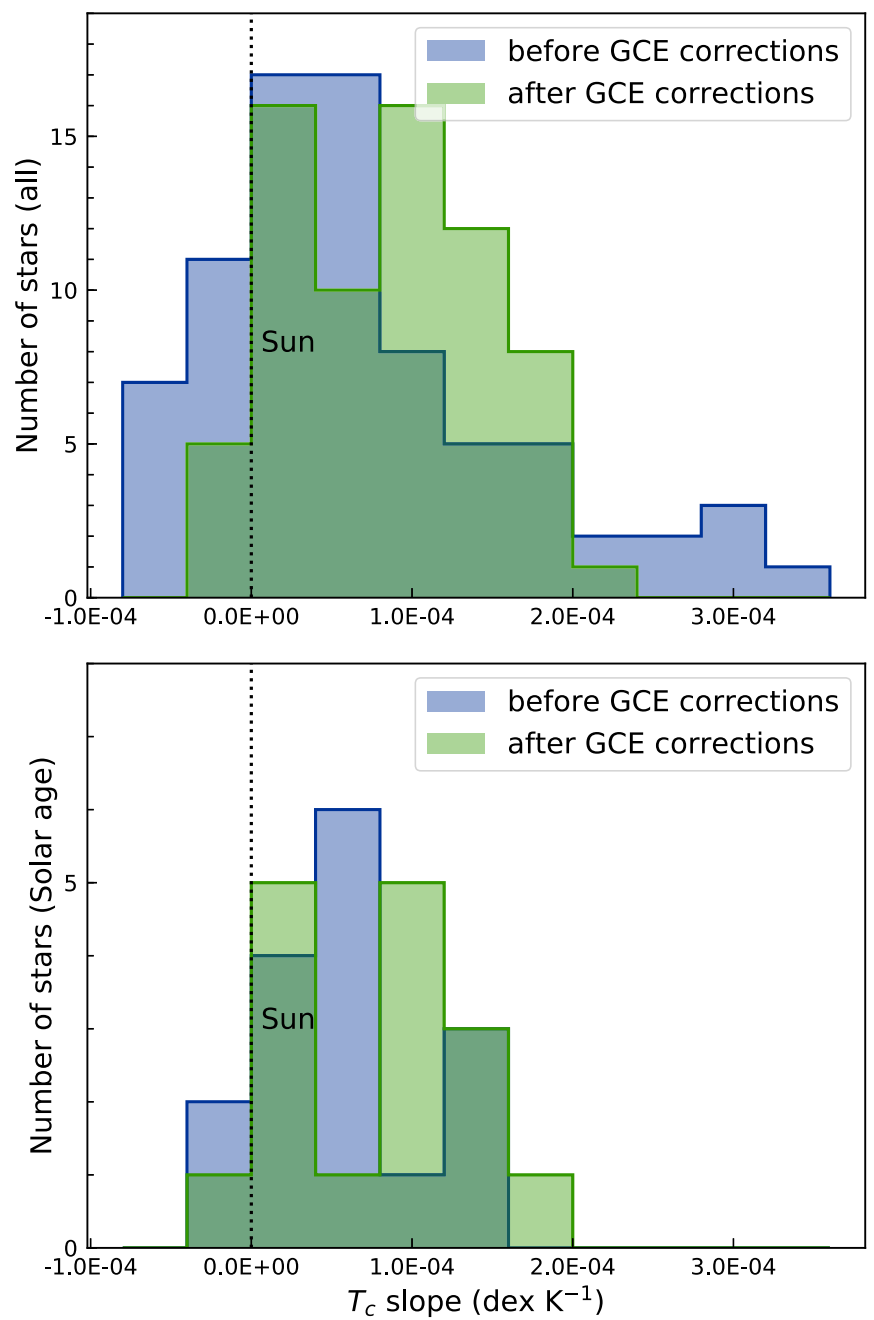

Figure 8. Distribution of $T_{\mathrm{C}}$ trends in the full solar twin sample (top) and in the subsample of twins with ages between 3.5 and 5.5 Gyr (bottom). Slopes in [X/Fe] against $T_{\mathrm{C}}$ are shown using abundances from before (blue) and after (green) the GCE corrections shown in Figure 3. The solar $T_{\mathrm{C}}$ slope, which is zero by definition, lies below $93 \%$ of the sample ( $84 \%$ at $2 \sigma$ confidence) after correcting for GCE. The Sun's relative deficiency in refractory material may be related to the rarity of the solar-system architecture among exoplanetary systems.

trends: in the alternative piecewise linear function, the Sun lies below $82 \%$ of the GCE-corrected sample.

As shown in the average abundances (Figure 5), the neutroncapture elements (including $\mathrm{Eu}, \mathrm{Pr}, \mathrm{Nd}$, and $\mathrm{Ce}$ ) tend to be the most discrepant from the $T_{\mathrm{C}}$ trend. This may be an indication that our GCE corrections, which are assumed to be simple linear relations with age, do not fully capture the intrinsic variance in abundance patterns. With a more complete marginalization over the abundance contributions of different nucleosynthetic processes, a sharper $T_{\mathrm{C}}$ trend with less scatter might emerge. This hypothesis is supported by the lower starto-star scatter in $T_{\mathrm{C}}$ slopes observed in the solar-age subsample (Figure 8, bottom panel).

\section{4. $\mathrm{T}_{C}$ Trends and Planets}

All of the stars analyzed in this work have been surveyed for planets with HARPS and in some cases HIRES, making them an excellent sample for investigating possible connections between $T_{\mathrm{C}}$ trends and planet formation. To do such an analysis 
properly, however, requires robust constraints on the occurrence rates of different planet types for the sample. Nonuniform time coverage of the RV observations and star-to-star differences in the manifestations of stellar variability across the sample make such occurrence studies difficult. In particular, terrestrial-mass planets are nearly impossible to detect (or rule out) given the current data. Future work on more robust methods of modeling stellar variability and extracting precise RVs may improve these prospects.

At present, there are no obvious conclusions to be drawn from the few solar twins that host candidate planets. HIP 11915 and HIP 5301, which host published Jupiter analogs (Naef et al. 2010; Bedell et al. 2015), both have $T_{\mathrm{C}}$ slopes of around (1.1-1.2) $\times 10^{-4}$ dex $\mathrm{K}^{-1}$ after GCE correction, near the sample average. The same is true of HIP 15527, which hosts a gas giant planet on an extremely eccentric orbit (Jones et al. 2006). HIP 68468, which hosts two close-in planet candidates and may have accreted planetary material in the past based on its anomalously high lithium abundance (Meléndez et al. 2017), has a Sun-like $T_{\mathrm{C}}$ trend relative to the full solar twin sample. Meanwhile, the radial velocity behavior of the most extreme $T_{\mathrm{C}}$ slope stars in the sample do not show any clear anomalies. Given the difficulty of detecting Earth-like planets and the number of confounding factors present in the $\mathrm{RVs}$, it is expected that any connection between terrestrial planet formation and detailed stellar composition should be extremely subtle and will require further analysis.

We leave a more sophisticated analysis of planet properties in this sample to the future. For the present, we merely note that the rarity of the Sun's abundance pattern and the apparent rarity of solar-system analogs (relative to the frequency of other types of planetary systems), when coupled with the interpretation of $T_{\mathrm{C}}$ trends as signatures of dust condensation, make the prospect of Sun-like abundance trends as a marker of solar-system-like planets worthy of further investigation.

\section{Conclusions}

Using our extremely high-precision measurements of 30 elemental abundances across 79 Sun-like stars in the solar neighborhood, we have constrained GCE trends, investigated the star-to-star variations in key planetary building blocks, and shown that the Sun has an unusual abundance trend relative to the majority of its twins.

The statistics of exoplanetary systems from the Kepler mission and radial velocity surveys have demonstrated that the architectures of most other planetary systems are dramatically different than our own, with approximately half of all Sun-like stars hosting likely volatile-rich planets with super-Earth radii in orbits smaller than that of Mercury (Rogers 2015; Winn \& Fabrycky 2015). The coincidence between the rarity of the Sun's abundance pattern and the solar-system architecture offers the intriguing possibility that stellar abundances can be used to identify systems harboring planets that are more like the Earth than the typical exoplanet. We look forward to more detailed exploration of the connections between stellar abundances and planetary system properties as the sample of bright, spectroscopy-friendly stars with well-characterized characterized planets vastly expands in the upcoming era of TESS, Gaia, and next-generation radial velocity spectrographs.

We thank the many scientists and engineers who made the HARPS observations possible. We also thank Fred Ciesla,
Jean-Michel Desert, Bruce Draine, David W. Hogg, Eliza Kempton, Boris Leistadt, Ben Montet, Melissa Ness, Poul Nissen, and the anonymous referee for valuable input. M.B. acknowledges support for this work from the NSF through the Graduate Research Fellowships Program (grant \#DGE1144082), the Josephine de Karman Fellowship Trust, the Illinois Space Grant Consortium, and the Lewis and Clark Fund for Exploration and Field Research in Astrobiology. J.L.B. acknowledges support for this work from the NSF (grant number AST-1313119), the Alfred P. Sloan Foundation, and the David and Lucile Packard Foundation. J.M. and L.S. acknowledge the support from FAPESP (2012/24392-2 and 2014/15706-9).

Facilities: ESO:3.6m (HARPS), Magellan:Clay (MIKE).

Software: numpy (van der Walt et al. 2011), matplotlib (Hunter 2007), IRAF (Tody 1986), MOOG (Sneden 1973), q2 (Ramírez et al. 2014), emcee (Foreman-Mackey et al. 2013).

\section{ORCID iDs}

Megan Bedell (i) https://orcid.org/0000-0001-9907-7742 Jacob L. Bean (iD https://orcid.org/0000-0003-4733-6532 Martin Asplund (iD https://orcid.org/0000-0002-5804-3682 TalaWanda Monroe (i) https://orcid.org/0000-0001-6797-6081 Luca Casagrande (iD https://orcid.org/0000-0003-2688-7511

\section{References}

Adibekyan, V., Delgado-Mena, E., Figueira, P., et al. 2016, A\&A, 591, A34 Adibekyan, V., Figueira, P., Santos, N. C., et al. 2015a, A\&A, 583, A94 Adibekyan, V., Santos, N. C., Figueira, P., et al. 2015b, A\&A, 581, L2 Adibekyan, V. Z., González Hernández, J. I., Delgado Mena, E., et al. 2014, A\&A, 564, L15

Adibekyan, V. Z., Sousa, S. G., Santos, N. C., et al. 2012, A\&A, 545, A32

Asplund, M., Grevesse, N., Sauval, A. J., \& Scott, P. 2009, ARA\&A, 47, 481

Baumann, P., Ramírez, I., Meléndez, J., Asplund, M., \& Lind, K. 2010, A\&A, 519, A87

Bedell, M., Meléndez, J., Bean, J. L., et al. 2014, ApJ, 795, 23

Bedell, M., Meléndez, J., Bean, J. L., et al. 2015, A\&A, 581, A34

Bensby, T., Feltzing, S., Gould, A., et al. 2017, A\&A, 605, A89

Bensby, T., Feltzing, S., \& Oey, M. S. 2014, A\&A, 562, A71

Bernstein, R., Shectman, S. A., Gunnels, S. M., Mochnacki, S., \& Athey, A. E. 2003, Proc. SPIE, 4841, 1694

Bond, J. C., O’Brien, D. P., \& Lauretta, D. S. 2010, ApJ, 715, 1050

Brewer, J. M., \& Fischer, D. A. 2016, ApJ, 831, 20

Burbidge, E. M., Burbidge, G. R., Fowler, W. A., \& Hoyle, F. 1957, RvMP, 29,547

Carter-Bond, J. C., O’Brien, D. P., Delgado Mena, E., et al. 2012, ApJL, 747, L2

Casagrande, L., Schönrich, R., Asplund, M., et al. 2011, A\&A, 530, A138

Castelli, F., \& Kurucz, R. L. 2004, arXiv:astro-ph/0405087

Chambers, J. E. 2010, ApJ, 724, 92

Delgado Mena, E., Israelian, G., González Hernández, J. I., et al. 2010, ApJ, 725,2349

Demarque, P., Woo, J.-H., Kim, Y.-C., \& Yi, S. K. 2004, ApJS, 155, 667

Dorn, C., Khan, A., Heng, K., et al. 2015, A\&A, 577, A83

dos Santos, L. A., Meléndez, J., Bedell, M., et al. 2017, MNRAS, 472, 3425

Dotter, A., Conroy, C., Cargile, P., \& Asplund, M. 2017, ApJ, 840, 99

Fischer, D. A., \& Valenti, J. 2005, ApJ, 622, 1102

Foreman-Mackey, D., Hogg, D. W., Lang, D., \& Goodman, J. 2013, PASP, 125,306

Fortney, J. J. 2012, ApJL, 747, L27

Gaia Collaboration, Brown, A. G. A., Vallenari, A., et al. 2016, A\&A, 595, A2 Gaidos, E. 2015, ApJ, 804, 40

Gilmore, G., Wyse, R. F. G., \& Kuijken, K. 1989, ARA\&A, 27, 555

Gonzalez, G. 1997, MNRAS, 285, 403

Gonzalez, G. 2011, MNRAS, 416, L80

Gonzalez, G., Carlson, M. K., \& Tobin, R. W. 2010, MNRAS, 407, 314

González Hernández, J. I., Delgado-Mena, E., Sousa, S. G., et al. 2013, A\&A, 552, A6 
González Hernández, J. I., Israelian, G., Santos, N. C., et al. 2010, ApJ, 720,1592

Hinkel, N. R., Young, P. A., Pagano, M. D., et al. 2016, ApJS, 226, 4

Høg, E., Fabricius, C., Makarov, V. V., et al. 2000, A\&A, 355, L27

Hogg, D. W., Bovy, J., \& Lang, D. 2010, arXiv:1008.4686

Hunter, J. D. 2007, CSE, 9, 90

Jones, H. R. A., Butler, R. P., Tinney, C. G., et al. 2006, MNRAS, 369, 249

Kharchenko, N. V. 2001, KFNT, 17, 409

Kobayashi, C., \& Nakasato, N. 2011, ApJ, 729, 16

Kuchner, M. J., \& Seager, S. 2005, arXiv:astro-ph/0504214

Lodders, K. 2003, ApJ, 591, 1220

Madhusudhan, N., Mousis, O., Johnson, T. V., \& Lunine, J. I. 2011, ApJ, 743, 191

Mayor, M., Pepe, F., Queloz, D., et al. 2003, Msngr, 114, 20

Meléndez, J., Asplund, M., Gustafsson, B., \& Yong, D. 2009, ApJL, 704, L66

Meléndez, J., Bean, J. L., Bedell, M., et al. 2015, Msngr, 161, 28

Meléndez, J., Bedell, M., Bean, J. L., et al. 2017, A\&A, 597, A34

Meléndez, J., \& Ramírez, I. 2007, ApJL, 669, L89

Naef, D., Mayor, M., Lo Curto, G., et al. 2010, A\&A, 523, A15

Nissen, P. E. 2015, A\&A, 579, A52

Nissen, P. E. 2016, A\&A, 593, A65

Öberg, K. I., Murray-Clay, R., \& Bergin, E. A. 2011, ApJL, 743, L16

Oh, S., Price-Whelan, A. M., Brewer, J. M., et al. 2018, ApJ, 854, 138

Önehag, A., Gustafsson, B., \& Korn, A. 2014, A\&A, 562, A102

Petigura, E. A., \& Marcy, G. W. 2011, ApJ, 735, 41

Ramírez, I., Allende Prieto, C., \& Lambert, D. L. 2013, ApJ, 764, 78

Ramírez, I., Asplund, M., Baumann, P., Meléndez, J., \& Bensby, T. 2010, A\&A, 521, A33
Ramírez, I., Meléndez, J., \& Asplund, M. 2009, A\&A, 508, L17

Ramírez, I., Meléndez, J., Bean, J., et al. 2014, A\&A, 572, A48

Ramírez, I., Meléndez, J., Cornejo, D., Roederer, I. U., \& Fish, J. R. 2011, ApJ, 740, 76

Rogers, L. A. 2015, ApJ, 801, 41

Santos, N. C., Adibekyan, V., Dorn, C., et al. 2017, A\&A, 608, A94

Santos, N. C., Adibekyan, V., Mordasini, C., et al. 2015, A\&A, 580, L13

Schuler, S. C., Flateau, D., Cunha, K., et al. 2011, ApJ, 732, 55

Sneden, C. A. 1973, PhD thesis, Univ. Texas

Sotin, C., Grasset, O., \& Mocquet, A. 2007, Icar, 191, 337

Spina, L., Meléndez, J., Karakas, A. I., et al. 2016a, A\&A, 593, A125

Spina, L., Meléndez, J., Karakas, A. I., et al. 2018, MNRAS, 474, 2580

Spina, L., Meléndez, J., \& Ramírez, I. 2016b, A\&A, 585, A152

Spina, L., Palla, F., Randich, S., et al. 2015, A\&A, 582, L6

Suárez-Andrés, L., Israelian, G., González Hernández, J. I., et al. 2018, A\&A, 614, A84

Teske, J. K., Khanal, S., \& Ramírez, I. 2016, ApJ, 819, 19

Thiabaud, A., Marboeuf, U., Alibert, Y., Leya, I., \& Mezger, K. 2015, A\&A, 580, A30

Tody, D. 1986, Proc. SPIE, 627, 733

Unterborn, C. T., Hull, S. D., Stixrude, L. P., et al. 2017, in Habitable Worlds 2017: A System Science Workshop (Houston, TX: LPI), 4034

Unterborn, C. T., Kabbes, J. E., Pigott, J. S., Reaman, D. M., \& Panero, W. R. 2014, ApJ, 793, 124

Unterborn, C. T., \& Panero, W. R. 2017, ApJ, 845, 61

Valenti, J. A., \& Fischer, D. A. 2005, ApJS, 159, 141

van der Walt, S., Colbert, S. C., \& Varoquaux, G. 2011, CSE, 13, 22

Winn, J. N., \& Fabrycky, D. C. 2015, ARA\&A, 53, 409 